\title{
Exploring the Lyapunov instability properties of high-dimensional atmospheric and climate models
}

\author{
Lesley De Cruz ${ }^{1}$, Sebastian Schubert ${ }^{2}$, Jonathan Demaeyer ${ }^{1}$, Valerio Lucarini ${ }^{2,3,4}$, and Stéphane Vannitsem ${ }^{1}$ \\ ${ }^{1}$ Royal Meteorological Institute of Belgium, Brussels, Belgium \\ ${ }^{2}$ Meteorological Institute, CEN, University of Hamburg, Hamburg, Germany \\ ${ }^{3}$ Department of Mathematics and Statistics, University of Reading, Reading, UK \\ ${ }^{4}$ Centre for the Mathematics of Planet Earth, University of Reading, Reading, UK
}

Correspondence: Lesley De Cruz (lesley.decruz@meteo.be)

Received: 22 December 2017 - Discussion started: 3 January 2018

Revised: 23 April 2018 - Accepted: 28 April 2018 - Published: 28 May 2018

\begin{abstract}
The stability properties of intermediate-order climate models are investigated by computing their Lyapunov exponents (LEs). The two models considered are PUMA (Portable University Model of the Atmosphere), a primitiveequation simple general circulation model, and MAOOAM (Modular Arbitrary-Order Ocean-Atmosphere Model), a quasi-geostrophic coupled ocean-atmosphere model on a $\beta$ plane. We wish to investigate the effect of the different levels of filtering on the instabilities and dynamics of the atmospheric flows. Moreover, we assess the impact of the oceanic coupling, the dissipation scheme, and the resolution on the spectra of LEs.
\end{abstract}

The PUMA Lyapunov spectrum is computed for two different values of the meridional temperature gradient defining the Newtonian forcing to the temperature field. The increase in the gradient gives rise to a higher baroclinicity and stronger instabilities, corresponding to a larger dimension of the unstable manifold and a larger first LE. The KaplanYorke dimension of the attractor increases as well. The convergence rate of the rate function for the large deviation law of the finite-time Lyapunov exponents (FTLEs) is fast for all exponents, which can be interpreted as resulting from the absence of a clear-cut atmospheric timescale separation in such a model.

The MAOOAM spectra show that the dominant atmospheric instability is correctly represented even at low resolutions. However, the dynamics of the central manifold, which is mostly associated with the ocean dynamics, is not fully resolved because of its associated long timescales, even at intermediate orders. As expected, increasing the mechanical atmosphere-ocean coupling coefficient or introducing a turbulent diffusion parametrisation reduces the Kaplan-Yorke dimension and Kolmogorov-Sinai entropy. In all considered configurations, we are not yet in the regime in which one can robustly define large deviation laws describing the statistics of the FTLEs.

This paper highlights the need to investigate the natural variability of the atmosphere-ocean coupled dynamics by associating rate of growth and decay of perturbations with the physical modes described using the formalism of the covariant Lyapunov vectors and considering long integrations in order to disentangle the dynamical processes occurring at all timescales.

\section{Introduction}

The dynamics of the atmosphere and the climate system is characterised by the property of sensitivity to initial states (Kalnay, 2003). This feature implies that any small errors in the initial conditions will progressively amplify until the forecast becomes useless, or in other words cannot be distinguished from any random state taken from the climatology of the system. This property was already recognised in the early developments of weather forecasts (Thompson, 1957) and was associated with the non-linear nature of deterministic dynamical systems by Lorenz (1963). These pioneering works sowed the seeds for the development of predictability theories for the atmosphere and climate, and for important progress in the context of dynamical systems, in partic- 
ular the development of chaos theory (Eckmann and Ruelle, 1985). This sensitivity property affects not only the dynamics of errors in the initial conditions, but also the errors that are present either in the model parametrisations or in the boundary conditions (Nicolis, 2007; Nicolis et al., 2009). Clarifying the nature of this sensitivity is therefore crucial in the perspective of improving forecasts at short, medium, and long term (Vannitsem, 2017).

The property of sensitivity to initial conditions in deterministic dynamical systems is often evaluated by computing the Lyapunov exponents that correspond to the asymptotic rates of amplification or decay of infinitesimally small perturbations, e.g. Eckmann and Ruelle (1985), Ott (2002), and Cencini et al. (2010). A system is chaotic if it possesses at least one positive Lyapunov exponent. Since the eighties many dynamical systems in various domains of science have been analysed from this perspective. This has revealed the presence of chaos in systems ranging from the fields of chemistry and biology to turbulence, e.g. Yamada and Ohkitani (1988), Gallez and Babloyantz (1991), Manneville (1995), and Sprott (2010). In the early days the investigations essentially dealt with low-order systems, but later the scope was broadened to include spatially distributed systems with a large number of degrees of freedom, in coupled maps, e.g. Nicolis et al. (1992), Vannitsem and Nicolis (1996), Cencini et al. (2010), and Yang and Radons (2013), and in partial differential equations, e.g. Manneville (1985, 1995), Vannitsem and Nicolis (1994), and Yang and Radons (2013). Recently, Lyapunov analysis was the subject of a special issue edited by Cencini and Ginelli (2013).

In parallel to these investigations in the context of basic sciences, several attempts to compute Lyapunov exponents in the context of meteorological and climate models have been made (see Vannitsem (2017)), in particular in intermediateorder atmospheric quasi-geostrophic models (with $\mathrm{O}(1000)$ variables) (Legras and Ghil, 1985; Vannitsem and Nicolis, 1997; Lucarini et al., 2007; Schubert and Lucarini, 2015, 2016). These analyses indicate that if realistic boundary conditions and forcings are imposed on the model under investigation, the number of positive exponents is high, which implies that the solution for the atmosphere lives on a highdimensional attractor. This suggests at first sight that the number of degrees of freedom needed to describe the dynamics is high and cannot be reduced to a low-order system.

However, the atmosphere cannot be treated as an autonomous system, as it interacts with other components of the climate system. These other components are characterised by longer timescales of motions. They are typically less intensely affected by some of the physical processes responsible for atmospheric instabilities, most notably convective and baroclinic instability. Moreover, the energetics of the atmosphere is mainly driven by thermodynamic processes that are dominated by the inhomogeneous absorption of solar radiation. The surface oceanic circulation, by contrast, is mostly mechanically driven by atmospheric winds (Lucarini et al.,
2014). On even longer timescales, buoyancy fluxes are an important driver for the deep ocean's thermohaline circulation.

This raises the question as to the impact of the coupling to other sub-domains of the climate system: are the other subdomains of the climate system stabilising the atmosphere or not? Vannitsem et al. (2015) partly addressed this question in the context of coupled low-order ocean-atmosphere systems. They found that the presence of the ocean and its exchanges (heat and momentum) with the atmosphere can drastically reduce the instability properties of the flow, confirming earlier results of Nese and Dutton (1993). As discussed below, the role of the ocean in modulating and impacting atmospheric instabilities is far from trivial.

Yet the problem of the predictability (in terms of Lyapunov instability) of the full-scale climate system including the different sub-domains is still open. Recently a new coupled ocean-atmosphere model was developed that could help answer key questions on the predictability properties of this type of system (De Cruz et al., 2016). The model was coined MAOOAM for Modular Arbitrary-Order Ocean-Atmosphere Model. The modular design of MAOOAM allows one to easily explore different model parameters and resolutions. In particular, the coupling strength between the ocean and the atmosphere should modify the predictability properties of the flow as illustrated in (Vannitsem et al., 2015). Moreover, the model resolution is also expected to play an important role in the instability properties of the flow as discussed in Lucarini et al. (2007) in the context of an atmospheric model.

\subsection{The properties of the tangent space}

As originally envisioned by Ruelle (1979), it is possible to associate with each Lyapunov exponent a corresponding infinitesimal perturbation that co-varies with the orbit that grows or decays asymptotically with the rate given by the corresponding exponent. These physical modes are usually referred to as covariant Lyapunov vectors (CLVs). The application of such a formalism to explore the properties of the tangent space was pioneered by Legras and Vautard (1995) and Trevisan and Pancotti (1998), before Ginelli et al. (2007) and Wolfe and Samelson (2007) provided efficient algorithms to compute them for high-dimensional systems. The CLVs have been used to study e.g. spatio-temporal chaos (Pazó et al., 2008, 2010), Rayleigh-Bénard convection (Xu and Paul, 2016), and the dynamics of the mid-latitude atmosphere in the quasi-geostrophic (QG) approximation (Schubert and Lucarini, 2015, 2016). Schubert and Lucarini (2015, 2016) have also underlined that CLVs allow for generalisation of the classic normal mode instability of fixed stationary states to the case of chaotic background state (e.g. Charney, 1947; Eady, 1949; Pedlosky, 1964) and allow for associating unstable/stable modes with specific paths of energy exchange and conversion. Trevisan et al. (2010) and Carrassi et al. (2008) also showed that performing data assimilation 
on the unstable manifold spanned by the CLVs corresponding to positive and neutral Lyapunov exponents is extremely efficient because it allows one to focus on the portion of the tangent space supporting the growth of errors.

Additionally, CLVs allow for understanding the properties of the tangent space and assess the hyperbolicity of the system, through the analysis of the statistics of the angles between the stable and unstable tangent manifolds across the attractor. These angles should always be bounded away from 0 or $\pi$ in the ideal case of uniform hyperbolicity. This point of view complements the investigation of the statistical properties of finite-time Lyapunov exponents (FTLEs): the probability density functions of the FTLEs whose long term averages correspond to positive exponents do not cross zero in the case of uniform hyperbolicity. Note that the uniform hyperbolicity is key to defining the structural stability of a chaotic dynamical system and provides, through the chaotic hypothesis by Gallavotti and Cohen (1995), an important working hypothesis for constructing the statistical mechanics of highdimensional chaotic systems, even in the case that such a system is not uniformly hyperbolic. Note that uniform hyperbolicity also allows for establishing a rigorous response theory for chaotic dynamical systems (Ruelle, 2009), which has also been shown to apply well in complex systems where there is no reason to believe that such stringent condition on the tangent space is obeyed; see, e.g., Lucarini et al. (2017).

\subsection{Multiscale properties}

As is well known, geophysical fluid dynamical (GFD) systems are characterised by relevant processes on multiple spatial and temporal scales of motion (Schneider, 2006; Vallis, 2006). These scales of motion can be isolated by assuming dominant dynamical balances and performing corresponding asymptotic expansions of the dynamical equations (Klein, 2010). A possible way to look at the signature of such a diverse range of dynamical processes in a nonlinear, chaotic setting can be found by considering the general idea proposed by Gallavotti (2014) according to which one can expect to find that LEs corresponding to smaller timescales are associated with CLVs characterised by small spatial scales. By looking at the properties of the structure of each CLV, one should ideally be able to understand what kind of dynamical processes (e.g. QG vs. mesoscale) are mainly responsible for such a physical mode.

The problem becomes particularly interesting when considering the coupling of two sub-domains with vastly different timescales, as done in the case of a low-order coupled ocean-atmosphere system in Vannitsem and Lucarini (2016). Three different manifolds were isolated in the model, the usual (highly) unstable manifold mainly associated with the dynamics of the atmosphere, a highly dissipative manifold also mainly associated with the dynamics of the atmosphere, and an extremely weakly (un-)stable manifold, that will be referred to here as the central manifold, essentially domi- nated by the dynamics and thermodynamics of the ocean but coupled to the atmosphere as well. The presence of a nontrivial central manifold is typical of the so-called partially hyperbolic systems (Pesin, 2004). The CLVs corresponding to the central manifold are geometrically quasi-degenerate, so that errors propagate easily between the various modes and impact both the atmosphere and the ocean. The corresponding FTLEs are strongly correlated and each have a rather slow decay of correlations, so that large deviation laws cannot be effectively estimated (Kifer, 1990; Touchette, 2009; Pazó et al., 2013; Laffargue et al., 2013). A particular consequence of this feature is that errors affecting the central manifold display a complex super-exponential behaviour. The question is therefore what the resolution of the coupled atmosphereocean model should be and what the time of observation should be such that a better separation emerges between such modes.

\subsection{Programmatic goals}

We wish to provide some first steps of a wider research programme aimed at performing a systematic investigation of the properties of the tangent space of GFD systems in a turbulent regime of motion. A first objective is to gain a better understanding of the multiscale properties of the dynamics and of the energy exchanges occurring across such scales. Furthermore, this programme aims at understanding the relevance of violations to the uniform hyperbolicity conditions in terms of predictability on different timescales, including the response - in a statistical mechanical sense - of the system to static and time-dependent perturbations.

In the present paper, we explore for the first time the Lyapunov spectra of the primitive-equation model, PUMA, and of intermediate-order configurations of the coupled oceanatmosphere system, MAOOAM. The first model is characterised by the presence of multiple scales of motions resulting from the fact that ageostrophic motions are not filtered, as opposed to the QG case (Klein, 2010). Instead, in the second model the multiscale properties come from the fact that the two represented geophysical fluids have largely different internal timescales. For PUMA, we consider the first 200 Lyapunov exponents for two different meridional temperature gradients. We study the properties of the Lyapunov spectrum and of the estimates of the Kaplan-Yorke dimension and Kolmogorov-Sinai entropy (Eckmann and Ruelle, 1985). In the case of MAOOAM, we investigate the role of dissipation introduced in the model (linear friction and effective diffusion) and the impact of the resolution of the models. For both models, the existence of large deviation laws of the FTLEs is tested.

In Sect. 2, the two models are described and Sect. 3 is devoted to a brief description of the Lyapunov instability analysis and the experimental set-ups. Section 4 summarises the results obtained so far and in Sect. 5 we present our future 
programme, which aims to clarify the instabilities of highresolution systems.

\section{Model description}

\subsection{PUMA}

The Portable University Model of the Atmosphere (PUMA) was introduced by Fraedrich et al. (1998). The intent of its developers was to design a model that gets close to state-ofthe-art circulation models and at the same time is still easy to use in teaching and research by single scientists. PUMA is the dynamical core of the Planet Simulator (PLASIM) which is a fully coupled climate model of intermediate complexity. PLASIM has been frequently used to study storm tracks (Fraedrich and Kirk, 2005), study tipping points (Lucarini et al., 2010; Boschi et al., 2013) or create large ensembles for climate change experiments allowing for interesting applications in economic modelling (Holden et al., 2014) or to assess the feasibility of linear response theory (Ragone et al., 2016; Lucarini et al., 2017).

Let us briefly summarise the equations of motion of PUMA and how the model is integrated in time. For further details we refer the reader to the PUMA User's Guide (Fraedrich et al., 1998). PUMA solves the primitive equations, which are derived from the Navier-Stokes equation on a rotating sphere by assuming approximate hydrostatic balance. This means that (convective) motions which are characterised by a vertical acceleration with a size comparable to gravity are filtered out (Holton, 2004). The prognostic equations as written in PUMA's code have four prognostic fields, the relative vorticity $\eta$, the divergence $D$, the logarithm of the surface pressure $\ln p_{\mathrm{s}}$, and the temperature $T$. These equations are

$$
\begin{aligned}
& \frac{\partial(\eta+f)}{\partial t}=\frac{1}{1-\mu^{2}} \frac{\partial F_{v}}{\partial \lambda}-\frac{\partial F_{u}}{\partial \mu}+P_{\eta} \\
& \frac{\partial D}{\partial t}=\frac{1}{1-\mu^{2}} \frac{\partial F_{u}}{\partial \lambda}-\frac{\partial F_{v}}{\partial \mu} \\
& -\left(\frac{U^{2}+V^{2}}{2\left(1-\mu^{2}\right)}+\Phi+T_{0} \ln p_{\mathrm{s}}\right)+P_{D} \\
& \frac{\partial p_{\mathrm{s}}}{\partial t}=-\int A \mathrm{~d} \sigma \\
& \frac{\partial T^{\prime}}{\partial t}=-\frac{1}{1-\mu^{2}} \frac{\partial\left(U T^{\prime}\right)}{\partial \lambda}-\frac{\partial\left(V T^{\prime}\right)}{\partial \mu}+D T^{\prime} \\
& -\dot{\sigma} \frac{\partial T}{\partial \sigma}+\kappa \frac{T}{p} \omega+\frac{J}{c_{p}}+P_{T}
\end{aligned}
$$

where the vorticity is defined as $\eta=\partial_{x} v-\partial_{y} u$ and the divergence is defined as $D=\partial_{x} u+\partial_{y} v$. Additionally, one takes into account the hydrostatic relation

$$
\frac{\partial \Phi}{\partial \ln \sigma}=-T
$$

and $T^{\prime}$ is defined as $T^{\prime}=T-T_{0}$ with $T_{0}=250 \mathrm{~K}$. Some abbreviations have been used:

$F_{u}=V(\eta+f)-\dot{\sigma} \frac{\partial U}{\partial \sigma}-T^{\prime} \frac{\partial \ln p_{\mathrm{s}}}{\partial \lambda}$

$F_{v}=-U(\eta+f)-\dot{\sigma} \frac{\partial V}{\partial \sigma}-T^{\prime}\left(1-\mu^{2}\right) \frac{\partial \ln p_{\mathrm{s}}}{\partial \mu}$

$A=D+\boldsymbol{V} \cdot \nabla \ln p_{\mathrm{s}}$

with $U=u \cos \phi$ and $V=v \cos \phi$. The variables used in this equation can be found in Table 1 .

PUMA is forced by Newtonian cooling which accounts in a crude yet effective way for the emission and the absorption of long- and short-wave radiation and for the heat convergence associated with convective processes (following Held and Suarez, 1994). This process is described by the equations

$$
\begin{aligned}
& \frac{J}{c_{p}}+P_{T}=\frac{T_{\mathrm{R}}(\phi, \sigma)-T}{\tau_{\mathrm{R}}}+H_{T}, \\
& T_{R}(\phi, \sigma)=T_{\mathrm{R}}^{\mathrm{vert}}(\sigma)+f(\sigma) T_{\mathrm{R}}^{\mathrm{hor}}(\phi),
\end{aligned}
$$

where $T_{\mathrm{R}}$ is the temperature restoration field that depends on the fixed meridional pole-to-Equator temperature gradient $\Delta T_{\mathrm{EP}}$ and the pole-to-pole gradient $\Delta T_{\mathrm{NS}}$. The latter gradient is zero in our experiments, so that we have equatorial symmetry in our boundary conditions and each solution we find is accompanied by a mirrored solution at the Equator. For completeness, we also add the full equations of the restoration field, and we refer the reader to Fraedrich et al. (1998) for a more detailed account:

$$
\begin{gathered}
T_{\mathrm{R}}^{\mathrm{hor}}(\phi)=\Delta T_{\mathrm{NS}} \frac{\sin (\phi)}{2}-\Delta T_{\mathrm{EP}}\left(\sin ^{2} \phi-\frac{1}{3}\right), \\
T_{\mathrm{R}}^{\mathrm{vert}}(\sigma)=\Delta T_{\mathrm{trop}}+\sqrt{\frac{L}{2}\left(z_{\mathrm{tp}}-z(\sigma)\right)^{2}+S^{2}} \\
\quad+\frac{L}{2}\left(z_{\mathrm{tp}}-z(\sigma)\right), \\
f(\sigma)= \begin{cases}\sin \left(\frac{\pi}{2}\left(\frac{\sigma-\sigma_{\mathrm{tp}}}{1-\sigma_{\mathrm{tp}}}\right)\right), & \text { if } \sigma \geq \sigma_{\mathrm{tp}} \\
0, & \text { if } \sigma<\sigma_{\mathrm{tp}} .\end{cases}
\end{gathered}
$$

Here, $\sigma_{\text {tp }}$ is the height of the tropopause, whereas $z_{\text {tp }}$ is the global constant height of the tropopause. $S$ is a technical smoothing parameter. Finally, the hyperdiffusion $H_{T}$ in Eq. (6) is defined as $H_{T}=\nabla^{8} T$ and parametrises small-scale interactions.

PUMA uses spherical harmonics and grid-point fields of the prognostic variables. Utilising the Fourier transform along the zonal direction and a Legendre transformation, PUMA computes the linear terms in spectral space and the non-linear terms in grid-point space. The time-stepping scheme is a combination of a leap-frog scheme with the Robert-Asselin filter.

The PUMA User's Guide includes more details and a complete description of the exact implementation and form of the various forcings (Fraedrich et al., 1998). 
Table 1. Symbols and variables in the PUMA equations.

\begin{tabular}{ll}
\hline Symbol & Description \\
\hline$T$ & temperature \\
$T_{0}$ & reference temperature \\
$T^{\prime}=T-T_{0}$ & temperature deviation from $T_{0}$ \\
$\eta$ & relative vorticity \\
$D$ & divergence \\
$p_{\mathrm{S}}$ & surface pressure \\
$\Phi$ & geopotential \\
$t$ & time \\
$\lambda, \phi$ & longitude, latitude \\
$\mu=\sin \phi$ & \\
$\sigma=\frac{p}{p_{\mathrm{s}}}$ & sigma vertical coordinate \\
$\dot{\sigma}=\frac{\mathrm{d} \sigma}{\mathrm{d} t}$ & vertical velocity in the $\sigma$-system \\
$\dot{p}=\frac{\mathrm{d} p}{\mathrm{~d} t}$ & vertical velocity in the $p$-system \\
$u, v$ & zonal, meridional component of horizontal \\
$V$ & velocity \\
$f$ & horizontal velocity with components $U, V$ \\
$J$ & Coriolis parameter \\
$c_{p}$ & diabatic heating rate \\
$\kappa$ & specific heat of dry air at constant pressure \\
\hline & adiabatic coefficient \\
\hline
\end{tabular}

\subsection{MAOOAM}

Although the atmospheric dynamics of both models are largely governed by the same processes, MAOOAM differs in many respects from the stand-alone PUMA model. Most importantly, the atmosphere of MAOOAM features both a mechanical and a thermal coupling with a shallowwater ocean layer, which is absent in PUMA. Furthermore, MAOOAM is a mid-latitude model which uses the quasigeostrophic approximation (Charney and Straus, 1980) on a $\beta$-plane (Vallis, 2006), whereas PUMA is a global primitiveequation model, in which the filtering is applied at a much smaller scale. The representation of the dynamical fields differs accordingly, with MAOOAM adopting a Fourier basis, using products of sine and cosine functions that respect the boundary conditions of a zonally periodic atmosphere over a rectangular ocean basin (De Cruz et al., 2016).

The dynamics of MAOOAM's two-layer atmosphere is described by the quasi-geostrophic vorticity equations, expressed in terms of the streamfunction fields $\psi_{\mathrm{a}}^{1}$ at $250 \mathrm{hPa}$ and $\psi_{\mathrm{a}}^{3}$ at $750 \mathrm{hPa}$ as in Charney and Straus (1980),

$$
\begin{aligned}
& \frac{\partial}{\partial t}\left(\nabla^{2} \psi_{\mathrm{a}}^{1}\right)+J\left(\psi_{\mathrm{a}}^{1}, \nabla^{2} \psi_{\mathrm{a}}^{1}\right)+\beta \frac{\partial \psi_{\mathrm{a}}^{1}}{\partial x} \\
& \quad=-k_{d}^{\prime} \nabla^{2}\left(\psi_{\mathrm{a}}^{1}-\psi_{\mathrm{a}}^{3}\right)+\frac{f_{0}}{\Delta p} \omega, \\
& \frac{\partial}{\partial t}\left(\nabla^{2} \psi_{\mathrm{a}}^{3}\right)+J\left(\psi_{\mathrm{a}}^{3}, \nabla^{2} \psi_{\mathrm{a}}^{3}\right)+\beta \frac{\partial \psi_{\mathrm{a}}^{3}}{\partial x} \\
& \quad=+k_{d}^{\prime} \nabla^{2}\left(\psi_{\mathrm{a}}^{1}-\psi_{\mathrm{a}}^{3}\right)-\frac{f_{0}}{\Delta p} \omega-k_{d} \nabla^{2}\left(\psi_{\mathrm{a}}^{3}-\psi_{\mathrm{o}}\right),
\end{aligned}
$$

in which the vertical velocity $\omega$ can be eliminated by applying the hydrostatic relation and the ideal gas law, as detailed in De Cruz et al. (2016).

Following Pierini (2011), the equation of motion for the ocean layer is described by

$$
\begin{aligned}
& \frac{\partial}{\partial t}\left(\nabla^{2} \psi_{\mathrm{o}}-\frac{\psi_{\mathrm{o}}}{L_{\mathrm{R}}^{2}}\right)+J\left(\psi_{\mathrm{o}}, \nabla^{2} \psi_{\mathrm{o}}\right)+\beta \frac{\partial \psi_{\mathrm{o}}}{\partial x} \\
& \quad=-r \nabla^{2} \psi_{\mathrm{o}}+\frac{C}{\rho h} \nabla^{2}\left(\psi_{\mathrm{a}}^{3}-\psi_{\mathrm{o}}\right) .
\end{aligned}
$$

The prognostic equations for the atmospheric and oceanic temperature fields, using an energy balance scheme as in Barsugli and Battisti (1998), are

$$
\begin{aligned}
& \gamma_{\mathrm{a}}\left(\frac{\partial T_{\mathrm{a}}}{\partial t}+J\left(\psi_{\mathrm{a}}, T_{\mathrm{a}}\right)-\sigma \omega \frac{p}{R}\right) \\
& \quad=-\lambda\left(T_{\mathrm{a}}-T_{\mathrm{o}}\right)+\epsilon_{\mathrm{a}} \sigma_{\mathrm{B}} T_{\mathrm{o}}^{4}-2 \epsilon_{\mathrm{a}} \sigma_{\mathrm{B}} T_{\mathrm{a}}^{4}+R_{\mathrm{a}}, \\
& \gamma_{\mathrm{o}}\left(\frac{\partial T_{\mathrm{o}}}{\partial t}+J\left(\psi_{\mathrm{o}}, T_{\mathrm{o}}\right)\right) \\
& \quad=-\lambda\left(T_{\mathrm{o}}-T_{\mathrm{a}}\right)-\sigma_{\mathrm{B}} T_{\mathrm{o}}^{4}+\epsilon_{\mathrm{a}} \sigma_{\mathrm{B}} T_{\mathrm{a}}^{4}+R_{\mathrm{o}} .
\end{aligned}
$$

The quartic terms in these equations are linearised by decomposing the temperature fields around a spatially and temporally constant equilibrium temperature, $T_{\mathrm{a}}=T_{\mathrm{a}}^{0}+\delta T_{\mathrm{a}}$ and $T_{\mathrm{o}}=T_{\mathrm{o}}^{0}+\delta T_{\mathrm{o}}$, and solving the quartic equation for the equilibrium temperature (Vannitsem et al., 2015).

The thermal wind relation allows one to link the atmospheric temperature anomaly $\delta T_{\mathrm{a}}$ to the baroclinic streamfunction $\theta_{\mathrm{a}} \equiv\left(\psi_{\mathrm{a}}^{1}-\psi_{\mathrm{a}}^{3}\right) / 2$, more specifically $\delta T_{\mathrm{a}}=$ $2 f_{0} \theta_{\mathrm{a}} / R$. Hence, the remaining independent dynamical fields are the barotropic atmospheric streamfunction field $\psi_{\mathrm{a}}$, defined as $\psi_{\mathrm{a}} \equiv\left(\psi_{\mathrm{a}}^{1}+\psi_{\mathrm{a}}^{3}\right) / 2$, the oceanic streamfunction field $\psi_{\mathrm{o}}$, and the temperature anomalies $\delta T_{\mathrm{a}}$ and $\delta T_{\mathrm{o}}$ of the atmosphere and the ocean. The other parameters and variables that feature in the MAOOAM equations are explained in Table 2.

The model equations are nondimensionalised, and the dynamical fields are expanded in a configurable set of Fourier modes. The MAOOAM code computes the coefficients for the resulting set of ordinary differential equations (ODEs) as algebraic formulae of the wavenumbers. These ODEs are then integrated using a fourth-order Runge-Kutta integration scheme. We refer the reader to De Cruz et al. (2016) for more details on the expansion of the dynamical fields in terms of Fourier modes, the computation of the coefficients, and the tensorial implementation of the ODEs.

In what follows, we will use a shorthand notation that uses the maximum wavenumbers $N_{x}$ and $N_{y}$ to specify the model resolution. If the resolution of the ocean and the atmosphere are the same, the model resolution is referred to as $N_{x} \times N_{y}$; otherwise, it is denoted as atm. $N_{x, \mathrm{a}} \times N_{y, \mathrm{a}}$, oc. $N_{x, \mathrm{o}} \times N_{y, \mathrm{o}}$ 
Table 2. Variables and parameters in the MAOOAM equations.

\begin{tabular}{|c|c|}
\hline Variable (units) & Description \\
\hline $\begin{array}{l}\psi_{\mathrm{o}}, \psi_{\mathrm{a}}\left(\mathrm{m}^{2} \mathrm{~s}^{-1}\right) \\
\omega=\mathrm{d} p / \mathrm{d} t\left(\operatorname{Pa~s}^{-1}\right) \\
T_{\mathrm{o}}, T_{\mathrm{a}}(\mathrm{K}) \\
\delta T_{\mathrm{o}}, \delta T_{\mathrm{a}}(\mathrm{K})\end{array}$ & $\begin{array}{l}\text { streamfunction of the ocean, atmosphere } \\
\text { vertical velocity in pressure coordinates } \\
\text { temperature of the ocean, atmosphere; } T_{x}=T_{x}^{0}+\delta T_{x} \\
\text { temperature anomaly of the ocean, atmosphere }\end{array}$ \\
\hline Parameter (units) & Description \\
\hline$n=2 L_{y} / L_{x}$ & meridional to zonal aspect ratio \\
\hline$L_{y}=\pi L(\mathrm{~km})$ & meridional extent of the domain \\
\hline$f_{0}\left(\mathrm{~s}^{-1}\right)$ & Coriolis parameter at $45^{\circ}$ latitude \\
\hline$\lambda\left(\mathrm{W} \mathrm{m}^{-2} \mathrm{~K}^{-1}\right)$ & heat transfer coefficient at the ocean-atmosphere interface \\
\hline$r\left(\mathrm{~s}^{-1}\right)$ & friction coefficient at the bottom of the ocean layer \\
\hline$C_{\mathrm{o}}, C_{\mathrm{a}}\left(\mathrm{W} \mathrm{m}^{-2}\right)$ & insolation coefficient of the ocean, atmosphere \\
\hline$k_{d}\left(\mathrm{~s}^{-1}\right)$ & friction coefficient at the ocean-atmosphere interface \\
\hline$k_{d}^{\prime}\left(\mathrm{s}^{-1}\right)$ & friction coefficient between the atmospheric layers \\
\hline$h(\mathrm{~m})$ & depth of the ocean layer \\
\hline$d=C /(\rho h)\left(\mathrm{s}^{-1}\right)$ & mechanical ocean-atmosphere coupling coefficient \\
\hline$R\left(\mathrm{~J} \mathrm{~kg}^{-1} \mathrm{~K}^{-1}\right)$ & gas constant of dry air \\
\hline$L_{\mathrm{R}}(\mathrm{km})$ & reduced Rossby deformation radius of the ocean \\
\hline$\rho\left(\mathrm{kg} \mathrm{m}^{-3}\right)$ & density of the ocean \\
\hline$\sigma_{\mathrm{B}}\left(\mathrm{W} \mathrm{m} \mathrm{m}^{2} \mathrm{~K}^{-4}\right)$ & Stefan-Boltzmann constant \\
\hline$\sigma\left(\mathrm{m}^{2} \mathrm{~s}^{-2} \mathrm{~Pa}^{-2}\right)$ & static stability of the atmosphere \\
\hline$\beta\left(\mathrm{m}^{-1} \mathrm{~s}^{-1}\right)$ & Rossby parameter $\frac{\mathrm{d} f}{\mathrm{~d} y}$ \\
\hline$\gamma_{\mathrm{o}}, \gamma_{\mathrm{a}}\left(\mathrm{J} \mathrm{m}^{-2} \mathrm{~K}^{-1}\right)$ & specific heat capacity of the ocean layer, atmosphere \\
\hline$T_{\mathrm{o}}^{0}, T_{\mathrm{a}}^{0}(\mathrm{~K})$ & constant solution for the temperature of the ocean, atmosphere \\
\hline$\epsilon_{\mathrm{a}}$ & grey-body atmosphere emissivity \\
\hline
\end{tabular}

\section{Methodology}

\subsection{Computation of the Lyapunov exponents}

Let us write the evolution laws of the autonomous system presented in Sect. 2 as a dynamical system:

$$
\frac{\mathrm{d} \boldsymbol{x}}{\mathrm{d} t}=\boldsymbol{f}(\boldsymbol{x}, \alpha)
$$

where $\boldsymbol{x}$ is a vector containing the entire set of relevant variables $\boldsymbol{x}=\left(x_{1}, \ldots, x_{N}\right)$ such as temperature or wind velocity, projected on a relevant set of modes as described in Sect. 2. The function $\boldsymbol{f}$ is a nonlinear function of the variables $\boldsymbol{x}$ and $\alpha$ represents a set of parameters.

Let us consider a small perturbation along the trajectory, $\boldsymbol{x}(t)$, generated by model (16), denoted $\delta \boldsymbol{x}(t)$. Provided that this perturbation is sufficiently small (ideally infinitely small), its dynamics can be described by the linearised equation,

$$
\frac{\mathrm{d} \delta \boldsymbol{x}}{\mathrm{d} t}=\left.\frac{\partial \boldsymbol{f}}{\partial \boldsymbol{x}}\right|_{\boldsymbol{x}(t)} \delta \boldsymbol{x}
$$

and a formal solution can be written as

$$
\delta \boldsymbol{x}(t)=\mathbf{M}\left(t, t_{0}\right) \delta \boldsymbol{x}\left(t_{0}\right)
$$

where the matrix $\mathbf{M}$ is referred to as the resolvent matrix or propagator. This matrix $\mathbf{M}$ is responsible for the amplification or contraction of the errors during the time period $t-t_{0}$. In order to get information independent of the initial or final time, a limit $\left(t-t_{0}\right) \rightarrow \infty$ should be taken. Oseledets $(1968,2008)$ demonstrates that provided that the system is ergodic, the following limit exists for almost all initial conditions $\boldsymbol{x}\left(t_{0}\right)=\boldsymbol{x}_{0}$,

$\lim _{t \rightarrow \infty}\left(\mathbf{M M}^{*}\right)^{1 / 2\left(t-t_{0}\right)}=\Lambda_{x_{0}}$

where $\mathbf{M}^{*}$ is the adjoint of $\mathbf{M}$. The backward Lyapunov exponents (Ershov and Potapov, 1998; Pazó et al., 2010) are then defined as the natural logarithm of the eigenvalues of $\Lambda_{x_{0}}$. These are usually represented in decreasing order and the full set of exponents is called the Lyapunov spectrum. Other definitions are available but will not be discussed here since we do not use them in this study. These can be found in Eckmann and Ruelle (1985) and Legras and Vautard (1995), and in a recent work in the context of the coupled ocean-atmosphere (Vannitsem and Lucarini, 2016).

If one or more LEs are positive, small errors on the initial conditions of the system grow exponentially and the system is chaotic. In that case, the time horizon of the system's predictability is proportional to the inverse of the largest Lya- 
punov exponent, $\frac{1}{\lambda_{1}}$. As this predictability horizon is expressed in days for operational forecasting, we also express the exponents $\lambda_{i}$ in units day ${ }^{-1}$. To translate the spectrum of LEs into spatial scales of the instabilities in an unambiguous way, the CLVs must also be determined. However, if there is scale-dependent dissipation, the largest negative LEs are most likely to be associated with the smallest, most dissipative scales.

The computation of the backward Lyapunov exponents follows the standard algorithm of (Shimada and Nagashima, 1979; Benettin et al., 1980) based on the Gram-Schmidt orthogonalisation.

1. An ensemble $\mathbf{E}$ of $N$ perturbation vectors is randomly initialised.

2. At every time step $t_{i}$, a matrix $\mathbf{P}_{i}$ that represents the linear propagator from $t_{i-1}$ to $t_{i}$ is computed using the tangent linear model along the model state trajectory. $\mathbf{P}_{i}$ is the equivalent of the matrix $\mathbf{M}$ for a finite time difference $t_{i}-t_{i-1}$. We take into account the numerical integration scheme when computing $\mathbf{P}_{i}$, by evaluating the model Jacobian at all intermediate points of the scheme. We have implemented the second- and fourth-order Runge-Kutta schemes, which require two and four evaluations of the Jacobian per time step, respectively.

3. As the model is integrated forward from time $t_{i}$ to $t_{i+b}$, the corresponding linear propagator $\mathbf{P}_{i, i+b}$ is approximated by multiplying the $b$ matrices, $\mathbf{P}_{i, i+b}=$ $\mathbf{P}_{i+b} \ldots \mathbf{P}_{i+1}$. In the experiments that follow, we have chosen $b=1$.

4. Every $b$ time steps, $\mathbf{E}$ is evolved with $\mathbf{P}_{i, i+b}$, and GramSchmidt orthogonalised (using a QR decomposition). The local Lyapunov spectrum is computed from the diagonal of $\mathbf{R}$.

5. Mean and variance of the local Lyapunov exponents are calculated.

The full Lyapunov spectrum of a model allows us to compute some additional interesting properties of its attractor. One of these is the Kaplan-Yorke or Lyapunov dimension $D_{\mathrm{KY}}$, which is an estimation of the information dimension $D_{1} . D_{1}$ is known to be less than or equal to the capacity or box-counting dimension $D_{0}$, also referred to as the fractal dimension (Frederickson et al., 1983). $D_{\mathrm{KY}}$ is defined as (Kaplan and Yorke, 1979)

$D_{\mathrm{KY}}=k+\frac{\lambda_{1}+\lambda_{2}+\ldots+\lambda_{k}}{\left|\lambda_{k+1}\right|}$,

where $k$ is the highest index for which the sum of the largest $k$ Lyapunov exponents is still strictly positive.

The second important property of the attractor is the Kolmogorov-Sinai or metric entropy $h_{\mathrm{KS}}$, a quantity that describes the rate of growth of the Shannon entropy (Eckmann and Ruelle, 1985; Boffetta et al., 2002), which characterises the quantity of information necessary to locate the solution on its attractor. Its upper bound is the sum of the positive Lyapunov exponents,

$h_{\mathrm{KS}} \leq \sum_{\lambda_{i}>0} \lambda_{i}$,

with the equality proven for a very particular class of systems, known as Axiom A systems. Here the KS entropy will be assumed to be close to the sum of positive exponents, and hence this sum will be referred to as the KS entropy.

\subsection{Large deviation laws}

Since the Lyapunov exponents are obtained by considering limiting conditions where the initial perturbations are very small and the time span over which the growth or decay rate is very long, they cannot reasonably be used to study predictability outside such conditions. FTLEs (Fujisaka, 1983; Abarbanel et al., 1991) have been proposed to address such shortcomings, with the caveat that they do not enjoy the extremely beneficial mathematical properties (especially norm independence) that characterise the Lyapunov exponents.

In this paper, we focus on the FTLEs and their relation to the asymptotic mean LEs. Hence, we are interested in averages $\sigma_{m}^{j}$ over a time $m$ of one backward Lyapunov exponent $\lambda_{j}$ and its statistics. As discussed in Schalge et al. (2013), Pazó et al. (2013), and Laffargue et al. (2013), some dynamical systems have the property that for a finite, but large $m$, the fluctuations of their FTLEs can be described by a large deviation law (Kifer, 1990; Touchette, 2009). This is the case for Axiom A systems, and invoking the chaotic hypothesis, extends to certain types of non Axiom A systems. For the finite-time backward LEs and for a large $m$, we will verify the following relation for the distribution $\mathcal{P}$ of the averages:

$\mathcal{P}\left(\sigma_{m}^{j}=x\right) \propto \exp \left(-m I_{j}(x)\right)$.

$I_{j}(x)$ is the rate function, which is independent of $m$. The rate function can be computed directly from this relation as

$I_{j}(x)=\lim _{m \rightarrow \infty}-\frac{1}{m} \log \left(\mathcal{P}\left(\sigma_{m}^{j}=x\right)\right)$.

If $x$ represents a time series, we have to take the autocorrelation into account. The FTLEs for the models under consideration have a non-zero autocorrelation. To account for this, the time series are decomposed into blocks that are decorrelated. For each LE, we find the smallest block size, called the decorrelation time $T_{\text {decorr }}$. The time $T_{\text {decorr }}$ is chosen to be the time lag when the autocorrelation drops below $1 / e$.

\subsection{Experimental design: PUMA}

We choose a simple set-up of PUMA. In this spirit, we also switch off orography. The system is forced via a constant 
temperature gradient between the Equator and the respective poles, as detailed in Sect. 2.1. We conduct simulations at a horizontal resolution of T42, which amounts to roughly $250 \mathrm{~km}$. In grid-point space this corresponds to a Gaussian grid with 64 latitudes and 128 longitudes. In the vertical direction we restrict the resolution to $10 \sigma$ levels. The integration scheme uses a time step of $1 \mathrm{~h}$.

The objective of our experiments with PUMA is to compute the backward Lyapunov exponents. For this we perform spin-up simulations for 30 years from random initial conditions. We then obtain the first 200 Lyapunov exponents using the Benettin algorithm described in Sect. 3.1. We allow the algorithm to converge for 5 years and finally obtain a time series of 25 years for all LEs. In order to explore two different chaotic regimes with many positive LEs, we perform two experiments with an Equator-to-pole temperature gradient $T_{\mathrm{EP}}$ of 50 and $60 \mathrm{~K}$, respectively (with $\Delta T_{\mathrm{NS}}=0$ ).

Note that in order to compute the Lyapunov exponents, it is necessary to construct the tangent linear of PUMA. We generated parts of the code using the program TAF by FastOpt (Giering and Kaminski, 2003).

\subsection{Experimental design: MAOOAM}

Table 3 lists the values of the physical parameters that are used in the present study. These are selected to lie within the realistic ranges previously derived by Vannitsem and De Cruz (2014), and correspond to the set-up used by De Cruz et al. (2016). In addition, we explore different values of the mechanical ocean-atmosphere coupling coefficient $d$ and the eddy viscosity coefficients $v_{\mathrm{a}}$ and $v_{\mathrm{o}}$, as well as a range of model resolutions.

All experiments are performed with the same integration parameters. The time step of 0.2 nondimensional time units corresponds to $32.3 \mathrm{~min}$ in dimensional units. Before calculating the Lyapunov spectrum, a transient run of $10^{8}$ nondimensional time units is performed, corresponding to 30726 years. Using the tangent linear model of MAOOAM, the backward Lyapunov exponents are then computed using the algorithm described in Sect. 3.1. In our simulations, the orthogonalisation is performed every time step, i.e. $b=1$. The Lyapunov spectrum is computed from simulations of 614 years.

The experiments are performed for different resolutions as discussed in Sect. 2 and for different dissipation schemes as described below.

- nodissip

This experiment corresponds to the set-up of De Cruz et al. (2016). In addition to the variables listed in Table 3 , the mechanical ocean-atmosphere coupling parameter $d$ is set to $1.1 \times 10^{-7} \mathrm{~s}^{-1}$.

- nodissip-reducedstress

For this "reduced-stress" experiment, the coupling parameter $d$ is reduced to $4 \times 10^{-8} \mathrm{~s}^{-1}$.
Table 3. Model parameter values that are identical across all MAOOAM configurations used in this study.

\begin{tabular}{lrlr}
\hline Parameter (unit) & Value & Parameter (unit) & Value \\
\hline$n=2 L_{y} / L_{x}$ & 1.5 & $L_{\mathrm{R}}(\mathrm{km})$ & 19.93 \\
$L_{y}=\pi L(\mathrm{~km})$ & $5.0 \times 10^{3}$ & $\rho\left(\mathrm{kg} \mathrm{m}^{-3}\right)$ & 1000 \\
$f_{0}\left(\mathrm{~s}^{-1}\right)$ & $1.032 \times 10^{-3}$ & $\sigma_{\mathrm{B}}\left(\mathrm{W} \mathrm{m}^{2} \mathrm{~K}^{-4}\right)$ & $5.6 \times 10^{-8}$ \\
$\lambda\left(\mathrm{W} \mathrm{m}^{-2} \mathrm{~K}^{-1}\right)$ & 15.06 & $\sigma\left(\mathrm{m}^{2} \mathrm{~s}^{-2} \mathrm{~Pa}^{-2}\right)$ & $2.16 \times 10^{-6}$ \\
$r\left(\mathrm{~s}^{-1}\right)$ & $1.0 \times 10^{-7}$ & $\beta\left(\mathrm{m}^{-1} \mathrm{~s}^{-1}\right)$ & $1.62 \times 10^{-11}$ \\
$C_{\mathrm{O}}\left(\mathrm{W} \mathrm{m}^{-2}\right)$ & 310 & $\gamma_{\mathrm{o}}\left(\mathrm{J} \mathrm{m}^{-2} \mathrm{~K}^{-1}\right)$ & $5.46 \times 10^{8}$ \\
$C_{\mathrm{a}}\left(\mathrm{W} \mathrm{m}^{-2}\right)$ & $C_{\mathrm{o}} / 3$ & $\gamma_{\mathrm{a}}\left(\mathrm{J} \mathrm{m}^{-2} \mathrm{~K}^{-1}\right)$ & $1.0 \times 10^{7}$ \\
$k_{d}\left(\mathrm{~s}^{-1}\right)$ & $3.0 \times 10^{-6}$ & $T_{\mathrm{a}}^{0}(\mathrm{~K})$ & 289.30 \\
$k_{d}^{\prime}\left(\mathrm{s}^{-1}\right)$ & $3.0 \times 10^{-6}$ & $T_{\mathrm{o}}^{0}(\mathrm{~K})$ & 301.46 \\
$h(\mathrm{~m})$ & 136.5 & $\epsilon_{\mathrm{a}}$ & 0.7 \\
$R\left(\mathrm{~J} \mathrm{~kg}^{-1} \mathrm{~K}^{-1}\right)$ & 287 & & \\
\hline
\end{tabular}

- dissipation

One of the physical processes that was not included in MAOOAM v1.0 (De Cruz et al., 2016) is the kinematic dissipation of energy due to turbulent diffusion, which becomes increasingly important at smaller spatial scales. This process is parametrised as a dissipation term in the prognostic equations for the atmospheric (barotropic) and oceanic streamfunction, which is proportional to the squared Laplacian of the respective streamfunction:

$$
\begin{aligned}
& D_{\mathrm{o}}=v_{\mathrm{o}} \nabla^{4} \psi_{\mathrm{o}}, \\
& D_{\mathrm{a}}=v_{\mathrm{a}} \nabla^{4} \psi_{\mathrm{a}} .
\end{aligned}
$$

We adopt the values for the parameters $v_{\mathrm{o}}$ and $v_{\mathrm{a}}$ from Van der Avoird et al. (2002), where they are estimated to be

$$
\begin{aligned}
& v_{\mathrm{o}}=1.76 \times 10^{4} \mathrm{~m}^{2} \mathrm{~s}^{-1}, \\
& v_{\mathrm{a}}=1 \times 10^{5} \mathrm{~m}^{2} \mathrm{~s}^{-1} .
\end{aligned}
$$

Furthermore, $d$ is set to $1.1 \times 10^{-7} \mathrm{~s}^{-1}$.

- dissipationx 10

In this experiment, $d=1.1 \times 10^{-7} \mathrm{~s}^{-1}$, but the parameters $v_{\mathrm{o}}$ and $v_{\mathrm{a}}$ are set to a higher value:

$$
\begin{aligned}
& v_{\mathrm{o}}=1.76 \times 10^{5} \mathrm{~m}^{2} \mathrm{~s}^{-1}, \\
& v_{\mathrm{a}}=1 \times 10^{6} \mathrm{~m}^{2} \mathrm{~s}^{-1} .
\end{aligned}
$$

- dissipation-reducedstress

This experiment has the same parameters as the "dissipation" experiment, except for the coupling parameter $d$ which is reduced to $4 \times 10^{-8} \mathrm{~s}^{-1}$.

Note that these idealised experiments do not take into account any dependence of the eddy (or turbulent) viscosity on the truncation scale, as is usually done in turbulence (e.g. 
Lesieur, 1990). However, even at the higher resolutions explored so far, we are still far from the scaling regimes for which these dependences may apply. In addition, the values of the eddy viscosity coefficients used are typically valid for a model configuration running at a spatial scale of the order of $100 \mathrm{~km}$ (Van der Avoird et al., 2002), which is smaller than the typical truncation used here. For this reason, we have performed a second experiment with a higher eddy viscosity coefficient. The problem of truncation and representation of subgrid-scale processes is an important open problem in climate modelling that needs careful attention. This matter falls beyond the scope of the present investigation, but forms the subject of a different study in the context of MAOOAM (Demaeyer and Vannitsem, 2016). Note that in principle the dissipated kinetic energy should become an input to the thermodynamic equations of the system as a positive heat source. As discussed in Lucarini and Fraedrich (2009), neglecting this process can have serious dynamical implications on long temporal scales. Additionally, an imperfect representation of this feedback between dynamics and thermodynamics is one of the sources of serious imperfections on the closure of the energy budget in climate models (Lucarini and Ragone, 2011; Lucarini et al., 2014). This issue will be analysed in future investigations.

\section{Results}

\subsection{PUMA}

Here we present the results for the two different experiments with PUMA, described in Sect. 3.3, and discuss our findings.

Figure 1 shows the 200 largest LEs of the two different Lyapunov spectra obtained in our experiments with PUMA. The averages were computed from a time series of 25 years of daily finite-time LEs. We can estimate the size of the attractor and by that estimate the degrees of freedom inherent to the attractor with the Kaplan-Yorke dimension $D_{\mathrm{KY}}$, as described in Sect. 3.1. The number of positive exponents and $D_{\mathrm{KY}}$ are shown in the caption of Fig. 1. Our findings confirm earlier results using two-layer QG models that suggested an increase in $D_{\mathrm{KY}}$ and the number of positive Lyapunov exponents for a higher meridional temperature gradient (Lucarini et al., 2007; Schubert and Lucarini, 2015).

There are two very small exponents since the model set-up is zonally symmetric, which in the limit of continuum creates an additional zero mode (see Schubert and Lucarini, 2015, for details). Otherwise, there are not many near-zero LEs in PUMA. There is continuity between the timescales that characterise the QG dynamics on the one hand, and faster, smaller-scale motions on the other hand. This shows that the usual assumption of a clear timescale separation adopted when applying the filtering to derive the QG equations is, in fact, rather stretched with respect to reality.

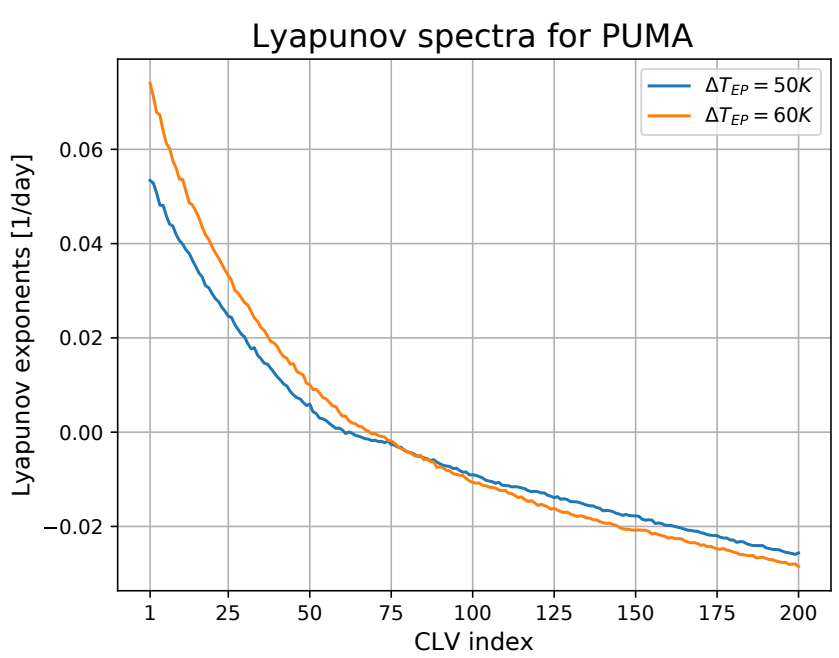

Figure 1. The 200 largest LEs of the Lyapunov spectra of PUMA for the two different set-ups with $\Delta T_{\mathrm{EP}}=50$ and $60 \mathrm{~K}$. For $\Delta T_{\mathrm{EP}}=$ $50 \mathrm{~K}$, the number of positive Lyapunov exponents is 61 and $D_{\mathrm{KY}}=$ 172.6. For $\Delta T_{\mathrm{EP}}=60 \mathrm{~K}$, the number of positive Lyapunov exponents is 68 and $D_{\mathrm{KY}}=187.0$.

Nevertheless, the $50 \mathrm{~K}$ spectrum in comparison to the $60 \mathrm{~K}$ spectrum has a smaller slope where the LE are near zero and negative. This may suggest the presence a longer term regime switching behaviour. One potential source for such a regime change is the switching between blocked and non-blocked states of the mid-latitudes atmosphere.

We have computed the blocking rate employing the wellestablished Tibaldi-Molteni Index (Tibaldi and Molteni, 1990). We indeed find a higher blocking rate for $50 \mathrm{~K}$ $(\approx 0.5 \%)$ than for $60 \mathrm{~K}(\approx 0.25 \%)$. We would like to explore this connection further in future studies, especially in the direction of studying the location of the CLVs during blocking (Schubert and Lucarini, 2016).

Next, the existence of a large deviation law for the FTLEs is verified, as described in Sect. 3.2. The decorrelation time $T_{\text {decorr }}$ is usually between 1 and 3 days. Therefore the rate function is computed for averages of length $t_{\text {ave }}=m \cdot 3$ days.

In Figs. 2 and 5 the results for the rate functions are shown for the fastest growing LE 1 (Fig. 2 and 3), fast-decaying LE 150 (Fig. 4 and 5), the positive and negative near-zero LEs 59 and 64 for $\Delta T_{\mathrm{EP}}=50 \mathrm{~K}$ (Fig. 6 and 7) and near-zero LEs 66 and 71 for $\Delta T_{\mathrm{EP}}=60 \mathrm{~K}$ (Fig. 8 and 9). Since Eq. (22) is needed to compute the rate function, a long time series is necessary to estimate the distribution $P$ reliably.

Our intent is to make at least a qualitative assessment of the convergence rate for $m \rightarrow \infty$. The top panels of these figures show the approximation of the respective distributions obtained via kernel density estimation (Scott, 1979) of the distribution of the block-averaged LEs. The bottom panels show the rate function for different $t_{\text {ave }}$ derived from Eq. (22). A detailed investigation of other metrics, such as the convergence of the variance, is provided in Appendix A. 
(a)

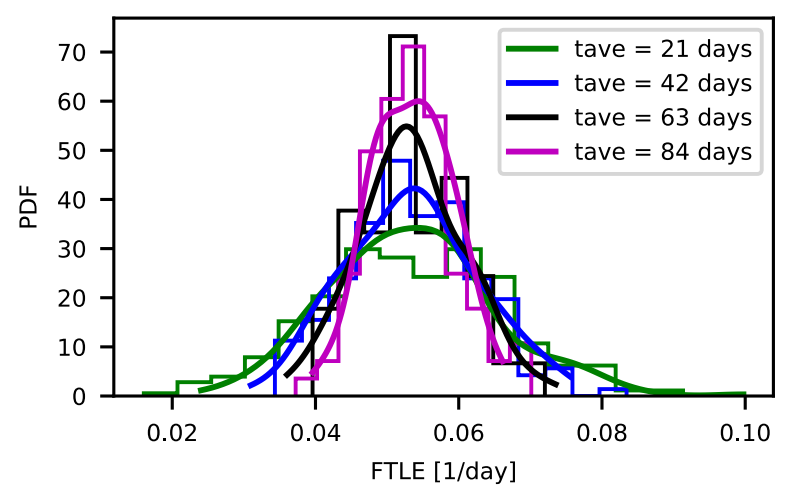

(b)

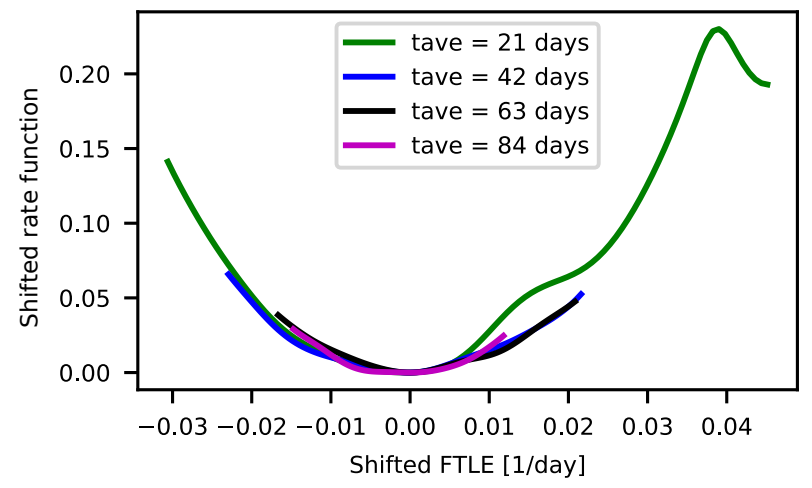

Figure 2. Distributions and rate functions of $\lambda_{1}$, the fastest-growing instability in PUMA, for $\Delta T_{\mathrm{EP}}=50 \mathrm{~K}$. Panel (a) shows the different distributions and their kernel-smoothing approximation of $\sigma_{m}^{1}$ where $t_{\text {ave }}$ is the respective $3 \mathrm{~m}$. Panel (b) shows a comparison of the rate functions, with the minimum moved to zero.

We make the following observations. The graphs suggest a convergence of the rate function for all LEs. Also, the rate functions' shape is approximately parabolic and the estimates of the rate functions appear to converge to the asymptotic with a comparable speed regardless of the value of the corresponding LE.

We interpret these results as stemming from the lack of a clear-cut timescale separation in a purely atmospheric model like PUMA. This is in opposition to what was originally speculated in Schubert and Lucarini (2015), where a primitive-equation model was expected to feature a timescale separation visible in the Lyapunov spectrum. Such a timescale separation would have been an a posteriori justification of the filtering by the QG approximation.

We have shown that in a primitive-equation model with a high-dimensional phase space of $\approx 60000$ the dimension of the attractor is small (order of 200) in comparison. Nevertheless, the unstable subspace can still be regarded as a highdimensional subspace with dimension of order of 50 . We also found sound results regarding the existence of a large deviation law independent of the growth rate of the linear perturba- (a)

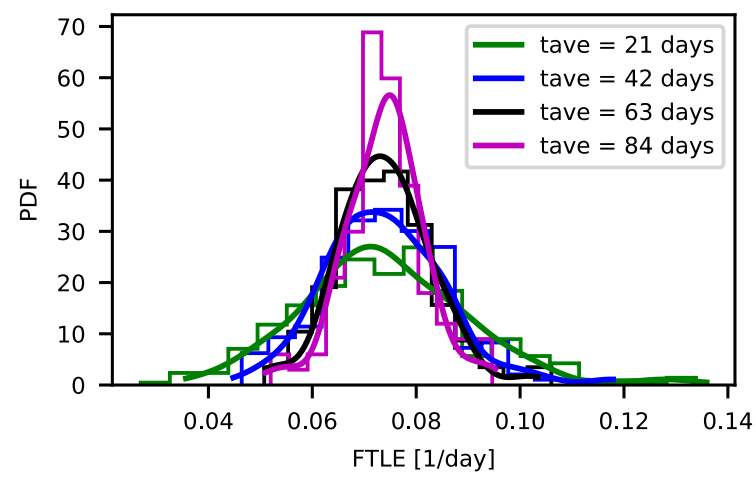

(b)

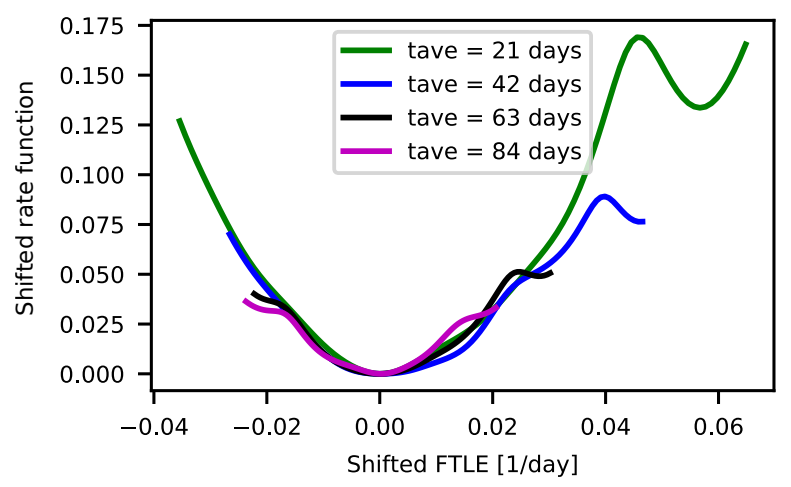

Figure 3. Distributions and rate functions of $\lambda_{1}$, the fastest-growing instability in PUMA, for $\Delta T_{\mathrm{EP}}=60 \mathrm{~K}$. Panels as in Fig. 2.

tions. In hindsight with respect to the findings in MAOOAM this can be explained with the absence of a clear timescale separation.

\subsection{MAООАМ}

The Lyapunov analysis is performed on the set of model configurations described in Sect. 3.4. Let us first evaluate the impact of the resolution on the amplitude of the dominant Lyapunov exponent. The largest Lyapunov exponent $\lambda_{1}$, which largely determines the limit of predictability, is plotted as a function of the model resolution for each experiment in Fig. 10. The dominant exponent $\lambda_{1}$ does not display a clear upward or downward trend versus model resolution and seems to stabilise for higher resolutions. This interesting feature suggests that the lower-order systems explored here already display a qualitatively correct amplitude for the dominant instability.

Furthermore, as we could expect, the predictability is enhanced for models where the scale-dependent dissipation term is present. The decrease in $\lambda_{1}$ also appears to suggest an enhanced predictability for models which have a larger ocean-atmosphere coupling parameter $d$, but this feature is not so clear for higher-resolution versions. Vannitsem (2017) studied the dependence of the predictability on this coupling 
(a)

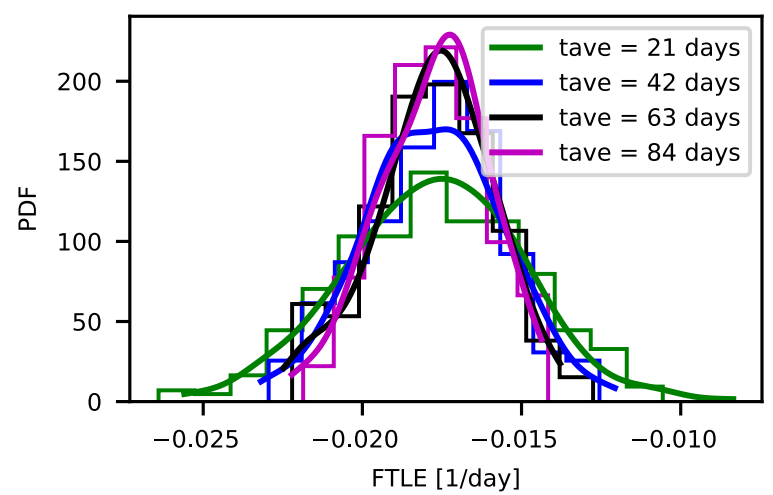

(b)

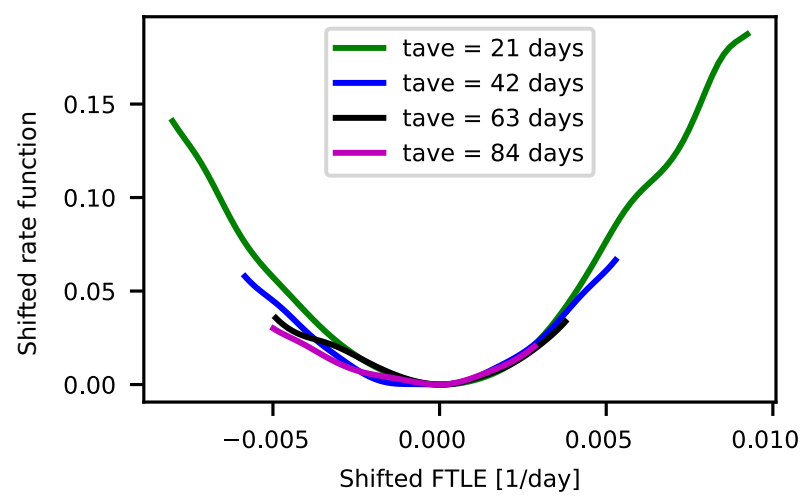

Figure 4. Distributions and rate functions of $\lambda_{150}$, a strongly decaying direction in PUMA, for $\Delta T_{\mathrm{EP}}=50 \mathrm{~K}$. Panels as in Fig. 2.

parameter in the low-order 36-variable model that forms the basis of MAOOAM. Two distinct mechanisms were identified to drive the increase in predictability with increasing $d$. To a first approximation, the mechanical coupling of the fast atmosphere to the slow ocean corresponds to an effective friction term which reduces error growth in the atmosphere. Moreover, increasing the ocean-atmosphere coupling above a critical value induces a sudden jump in predictability, associated with the development of a slow coupled oceanatmosphere mode (Vannitsem et al., 2015; Vannitsem, 2017).

Figures 11 to 14 show the full sets of Lyapunov exponents, or Lyapunov spectra, for the different experiments. These figures reveal the presence of three ranges in the spectrum of Lyapunov exponents: the positive, negative nearzero, and large-amplitude negative Lyapunov exponents, associated with the unstable, central, and stable manifolds, respectively, in qualitative agreement with what was found in Vannitsem and Lucarini (2016). We expect that the stable and unstable manifolds mainly characterise the dissipative and unstable motions of the atmosphere, while the central manifold also projects considerably on the variables of the ocean.

The highly populated central manifold of MAOOAM is in stark contrast to the few near-zero LEs in PUMA. Be- (a)

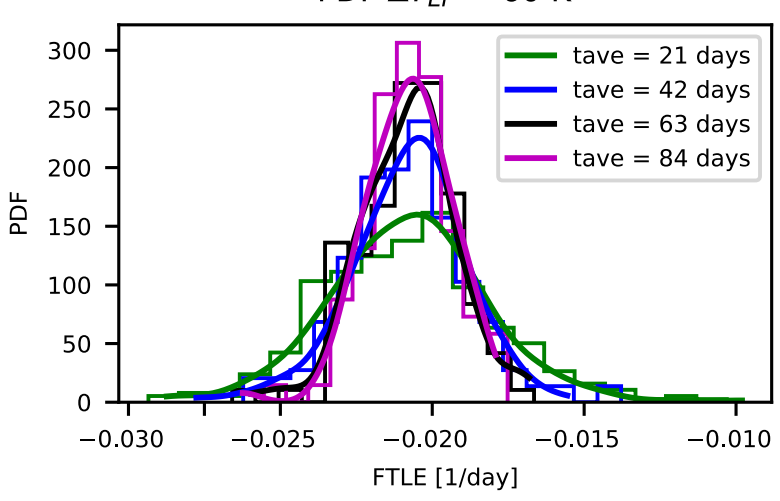

(b)

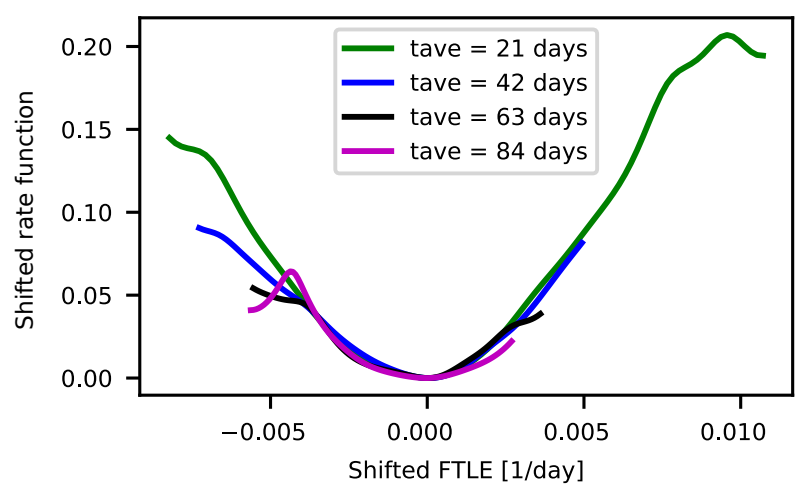

Figure 5. Distributions and rate functions of $\lambda_{150}$, a strongly decaying direction in PUMA, for $\Delta T_{\mathrm{EP}}=60 \mathrm{~K}$. Panels as in Fig. 2.

ing a purely atmospheric model, PUMA's Lyapunov spectrum does not exhibit the large timescale separation present in MAOOAM. Indeed, the spectrum of PUMA bears more resemblance to that of the QG two-layer model of Schubert and Lucarini (2015).

Upon increasing the number of modes in the ocean and the atmosphere, the number of positive Lyapunov exponents (indicated with a vertical arrow) consistently increases, but not as much as the number of strongly negative exponents. This suggests that most of the additional spatial scales that are resolved by the higher-resolution models are highly dissipative, hence increasing the number of strongly negative Lyapunov exponents. The additional positive and near-zero exponents that are introduced at these scales nevertheless indicate that the added resolution still resolves some scales that are important for the description of the dynamics. This is in agreement with the conclusion in De Cruz et al. (2016), where it was shown that in order to describe the ocean dynamics, one needs to be able to resolve the Rhines scale $L_{R h}=\sqrt{\frac{U}{\beta}}$, requiring oceanic wavenumbers as high as of 40-50.

Figure 15 plots the Kaplan-Yorke dimension $D_{\mathrm{KY}}$ as a function of the model resolution. This shows that $D_{\mathrm{KY}}$ is the highest for the models that do not include the scale- 
(a)

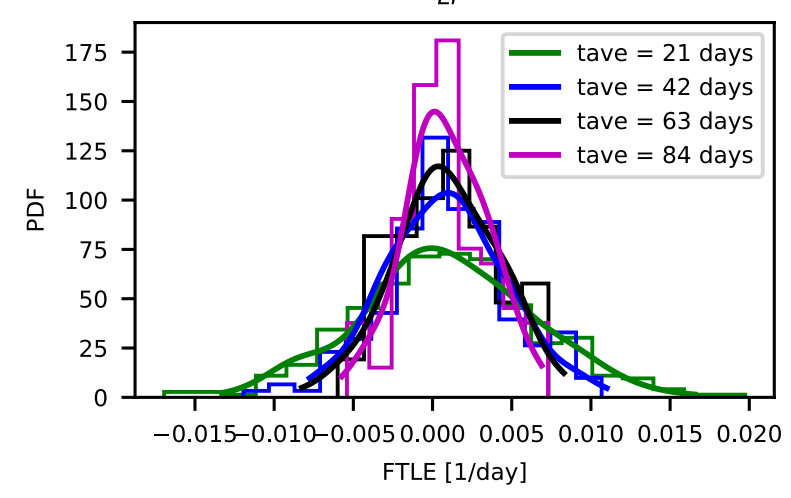

(b)

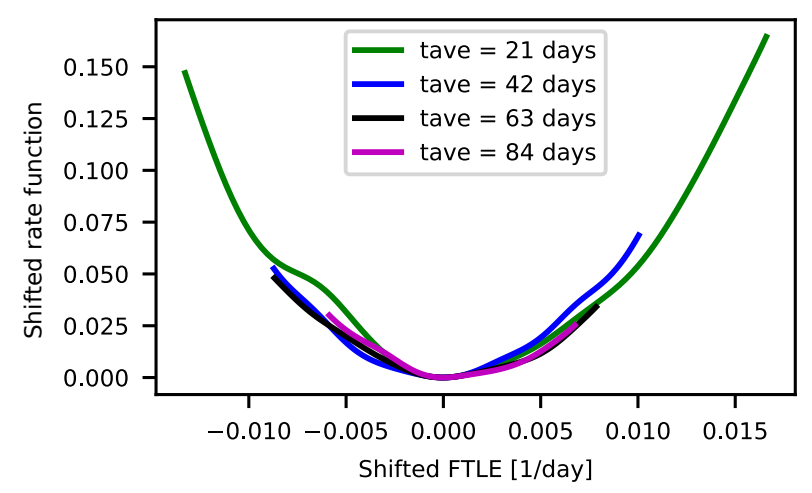

Figure 6. Distributions and rate functions of $\lambda_{59}$, a near-zero, growing instability in PUMA, for $\Delta T_{\mathrm{EP}}=50 \mathrm{~K}$. Panels as in Fig. 2.

dependent dissipation process (nodissip). A reduction in the ocean-atmosphere coupling $d$ appears to slightly increase $D_{\mathrm{KY}}$ for most model resolutions, both in the case with and without scale-dependent dissipation. The 10 -fold increase in the dissipation parameters $v_{\mathrm{o}}$ and $v_{\mathrm{a}}$ (dissipationx 10) results in the lowest values for $D_{\mathrm{KY}}$, as can be expected from a more dissipative but still chaotic system.

As the number of dimensions increases quadratically and not linearly for the consecutive model resolutions, it is instructive to rescale $D_{\mathrm{KY}}$ by the number of dimensions $N$, as shown in Fig. 16. This shows that while $D_{\mathrm{KY}}$ increases with resolution, the attractor dimension's fraction of the full phase space dimension decreases (even if slowly) with increasing resolution from the atm. $6 \times 6$, oc. $6 \times 6$ models onward, for all experiments, suggesting that one is adding in higher proportion highly stable modes that do not necessarily play an important role in the dynamics. In other words, we are not in the regime where the system is extensive, as, in fact, the geometry of the domain is fixed and we are capturing a larger and larger (yet insufficient) fraction of the active dynamical processes as the resolution is increased. Had we reached the optimal resolution, Fig. 15 would be flat, and Fig. 16 would approach zero. (a)

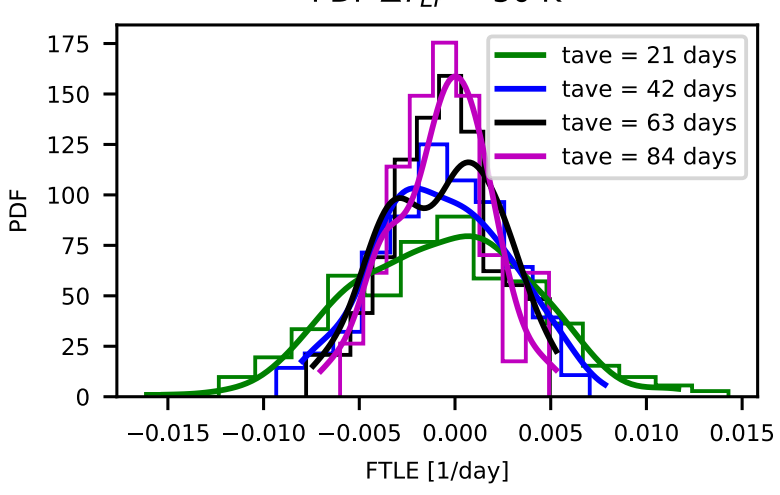

(b)

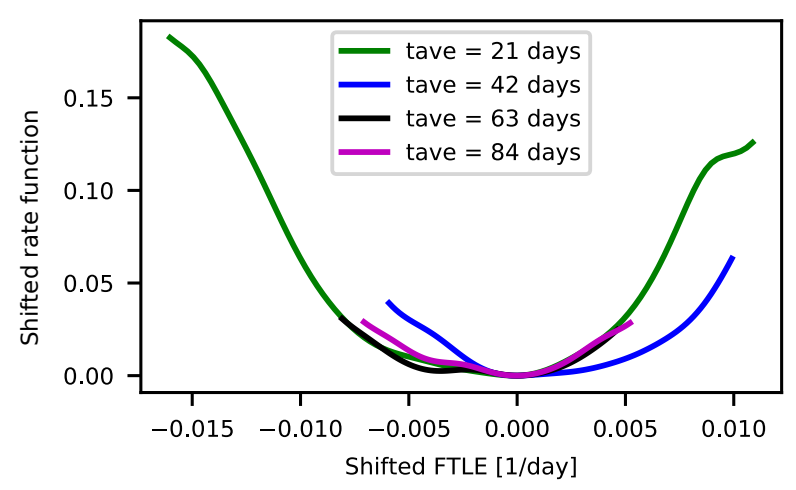

Figure 7. Distributions and rate functions of $\lambda_{64}$, a near-zero, decaying instability in PUMA, for $\Delta T_{\mathrm{EP}}=50 \mathrm{~K}$. Panels as in Fig. 2 .

Figure 17 shows the Kolmogorov-Sinai entropy $h_{\mathrm{KS}}$ versus model resolution, for the different experiments. The trends for the "nodissip" and "nodissip-reducedstress" experiments appear to suggest that $h_{\mathrm{KS}}$ would increase unboundedly for increasing model resolution if a parametrisation for the scale-dependent dissipation is absent. The experiments that take this process into account paint a more realistic picture, with $h_{\mathrm{KS}}$ levelling off at the highest model resolutions.

An additional experiment is performed by increasing the resolution of the ocean and of the atmosphere separately starting from a specific symmetric configuration " $6 \times 6$ ". Figure 18 displays the Lyapunov spectra for model configuration "dissipation". Two important features stand out: (i) when the resolution of the atmosphere is increased, the majority of the new exponents populate the stable manifold; (ii) by contrast, when the resolution of the ocean is increased, the number of slightly positive and slightly negative exponents increases considerably. This also suggests that the increase in Lyapunov dimension and the number of positive exponents after a resolution of atm. $6 \times 6-$ oc. $6 \times 6$ should be attributed to the presence of the ocean. In this sense the ocean plays an active role in the development of the coupled dynamics. Indeed, the quantity $D_{\mathrm{KY}} / N$, which approximates the relative fraction of the attractor's dimension, increases for 
(a)
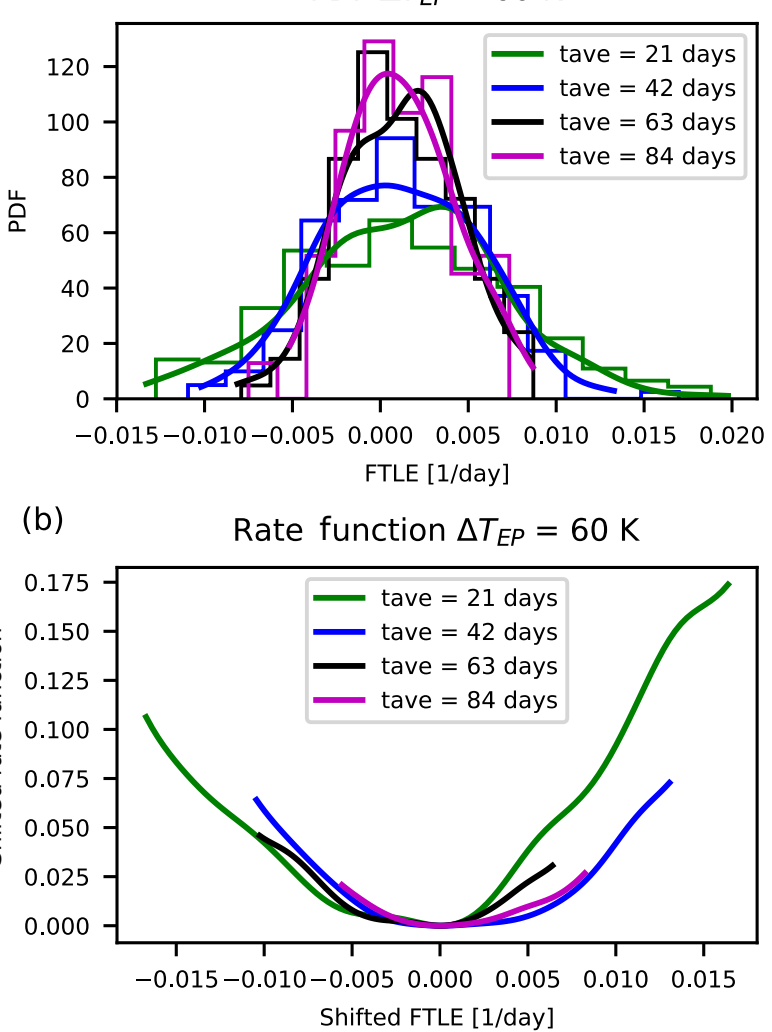

Figure 8. Distributions and rate functions of $\lambda_{66}$, a near-zero, growing instability in PUMA, for $\Delta T_{\mathrm{EP}}=60 \mathrm{~K}$. Panels as in Fig. 2.

increasing ocean resolution, but decreases for increasing atmosphere resolution, as illustrated in Fig. 19. This result deserves an extensive investigation by looking at the properties of the CLVs.

As a final analysis, we have given a preliminary look at whether large deviation laws can be established for the longterm statistics of the FTLEs. In what follows, we consider the " $9 \times 9$ " simulations. Similarly to what was found in a previous analysis performed on a severely truncated version of MAOOAM (Vannitsem and Lucarini, 2016), we find that the time series of the FTLEs corresponding to the strongly damped modes are weakly correlated. This would suggest that one can construct the rate functions defining the large deviations laws. The rate functions are shown in Fig. 20a-c for the 351st LE. Their convergence properties are investigated in Appendix A2, and indicate that we have not yet converged to the central limit theorem even for these strongly damped modes. Additionally, the lagged time correlation of the nearzero LEs are very strong and it makes no sense to look for large deviation laws in this case.

In contrast to what was presented in Vannitsem and Lucarini (2016), establishing large deviation laws for the FTLEs associated with positive LEs is not trivial, even when one considers the first FTLE. Lagged-time correla-
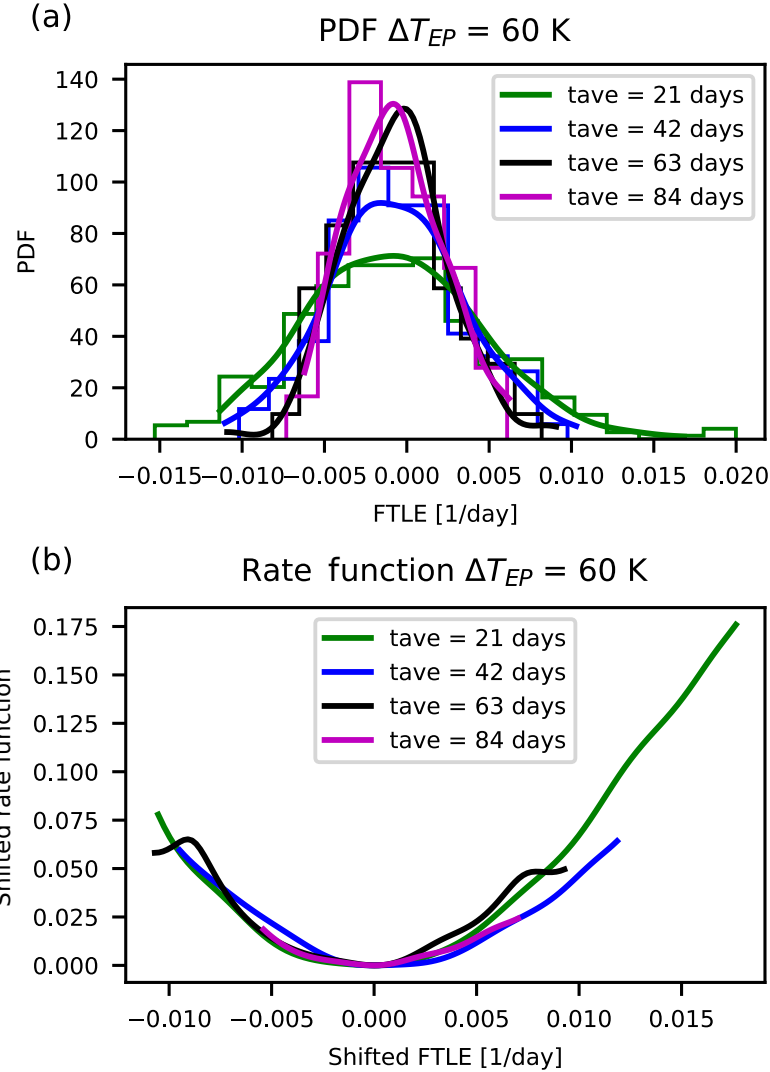

Figure 9. Distributions and rate functions of $\lambda_{71}$ a near-zero, decaying instability in PUMA, for $\Delta T_{\mathrm{EP}}=60 \mathrm{~K}$. Panels as in Fig. 2.

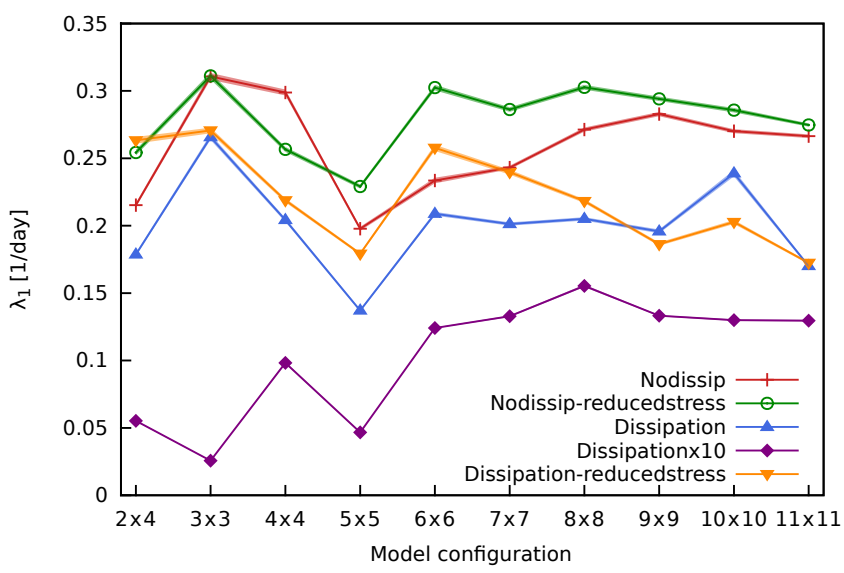

Figure 10. The largest Lyapunov exponent $\lambda_{1}$ of MAOOAM as a function of the model resolution for the different experiments: nodissip (red pluses), nodissip-reducedstress (green circles), dissipation (blue upward-pointing triangles) dissipationx10 (purple diamonds), dissipation-reducedstress (orange downward-pointing triangles).

tions are such that the available time series are not sufficiently long to reach the asymptotic limit. This is even the case in the nodissip simulation scenario, despite the larger 


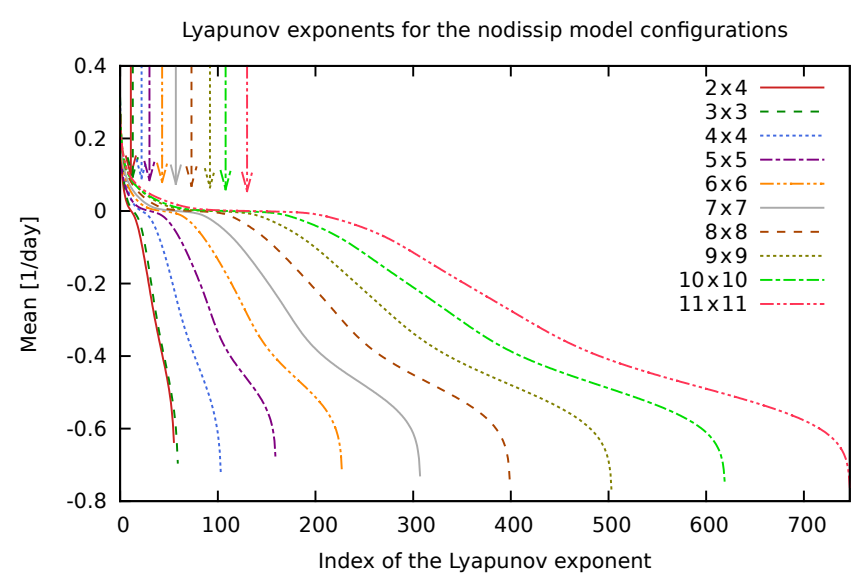

Figure 11. Lyapunov spectra of MAOOAM for the "nodissip" experiment, for model configurations from atm. $2 \times 4$, oc. $2 \times 4$ (red full line) up to atm. $11 \times 11$, oc. $11 \times 11$ (pink dash-dot-dotted line). Lyapunov exponents are ranked in decreasing order, and the index of the smallest positive Lyapunov exponent is indicated with a downward-pointing arrow for each model configuration.

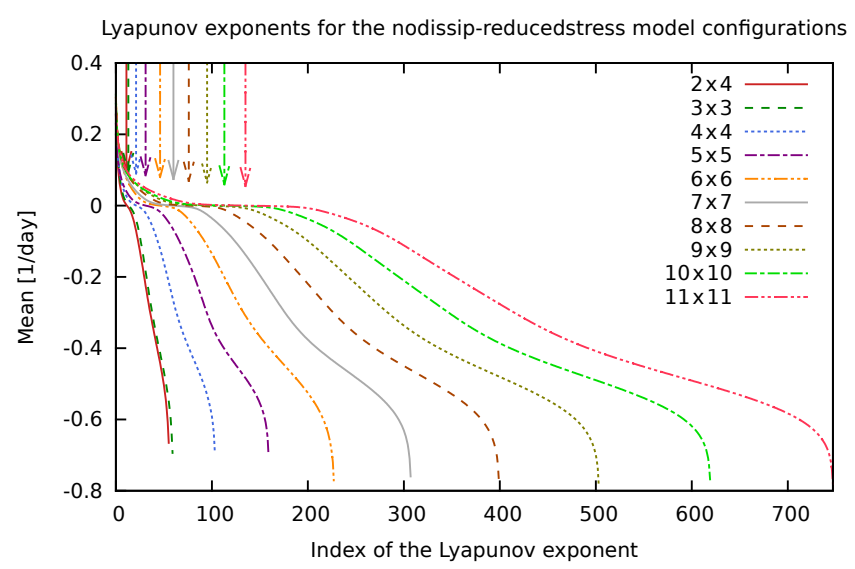

Figure 12. Lyapunov spectra of MAOOAM for the "nodissipreducedstress" experiment. Colours and arrows as in Fig. 11.

value of the first LE and faster decay of correlations; compare Fig. 21a-c. This suggests that when many unstable modes are present, disentangling their long-term properties requires very long integrations, possibly as a result of geometrical quasi-degeneracies among such modes. This is an issue that should be further explored, given its practical and theoretical relevance. One can conjecture that the damped modes do not feature such properties as their dynamics is mostly driven by linear dissipative processes. Therefore, we propose that an accurate analysis of the tangent space with the formalism of CLVs is required to advance our understanding of predictability at medium and long timescales.

In brief, these results indicate that the dominant instabilities of the coupled ocean-atmosphere system are well captured by MAOOAM, even at low resolutions. However, the

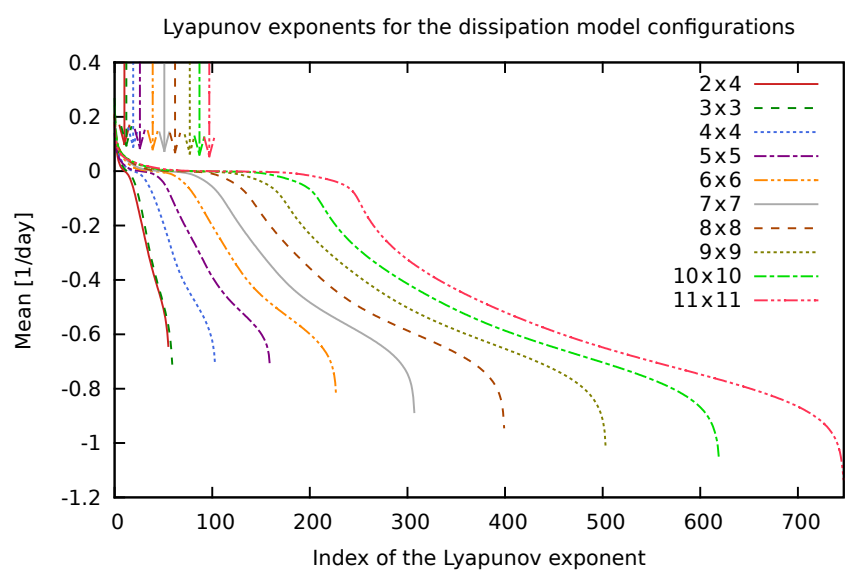

Figure 13. Lyapunov spectra of MAOOAM for the "dissipation" experiment. Colours and arrows as in Fig. 11.

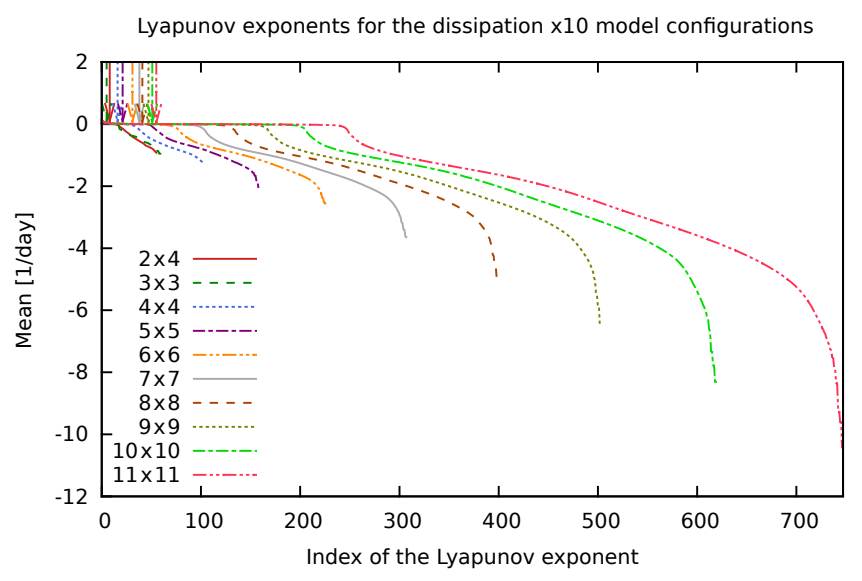

Figure 14. Lyapunov spectra of MAOOAM for the "dissipationx10" experiment. Colours and arrows as in Fig. 11.

increase in the Lyapunov dimension with the resolution implies that the relevant dynamics of the system are not yet fully resolved, in agreement with De Cruz et al. (2016). The main role of the ocean in this matter is confirmed by varying the ocean and atmosphere resolutions independently. Conversely, increasing the resolution of the atmosphere only adds highly dissipative modes. Finally, in contrast with what was found for a low-order version of MAOOAM (Vannitsem and Lucarini, 2016), large deviation laws cannot be established for the near-zero and positive FTLEs in the " $9 \times 9$ " configuration.

\section{Toward a new programme}

The chaotic nature of the atmosphere and of the climate system has been investigated in the present work in the context of a primitive-equation atmospheric model and a coupled ocean-atmosphere model. Both systems suggest that high- 


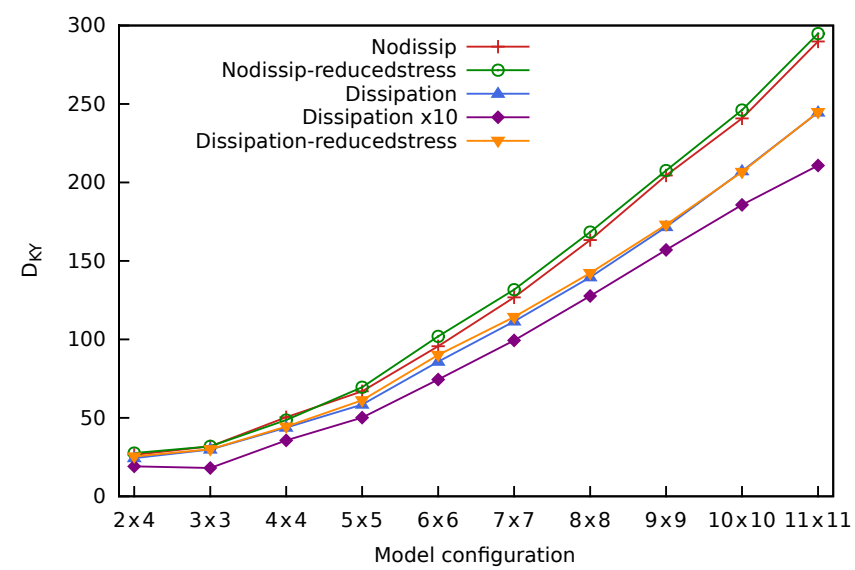

Figure 15. Kaplan-Yorke or Lyapunov dimension $D_{\mathrm{KY}}$ of MAOOAM as a function of the resolution for the different experiments. Colours as in Fig. 10.

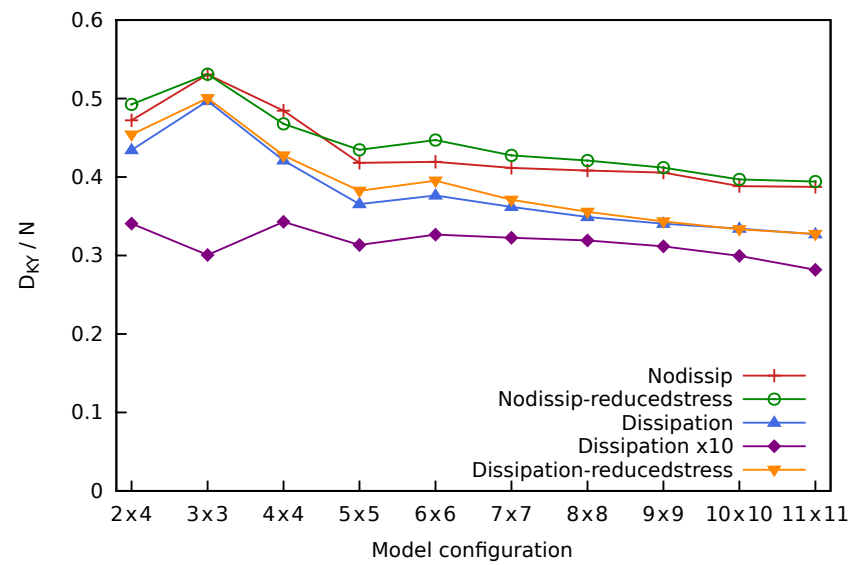

Figure 16. Kaplan-Yorke or Lyapunov dimension $D_{\mathrm{KY}}$ of MAOOAM divided by the total number of dimensions $N$, as a function of the resolution for the different experiments. Colours as in Fig. 10.

dimensional dynamical processes are at play with very interesting distinct specificities.

The Lyapunov spectra of the two models considered here have rather different qualitative features, as a result of their structural differences, which have profound impacts on the type of possible instability mechanisms. Following Gallavotti and Lucarini (2014), one expects that if a clear timescale separation between distinct dynamical regimes is present, one should find that the Lyapunov exponents can be divided into separate groups, corresponding to distinct clusters in their values. This is the analogue in full nonlinear terms of what is envisioned by the usual scale analysis of GFD equations.

MAOOAM is a coupled quasi-geostrophic atmosphereocean model, which, by definition, features a large timescale separation between ocean and atmosphere, and lacks a satis-

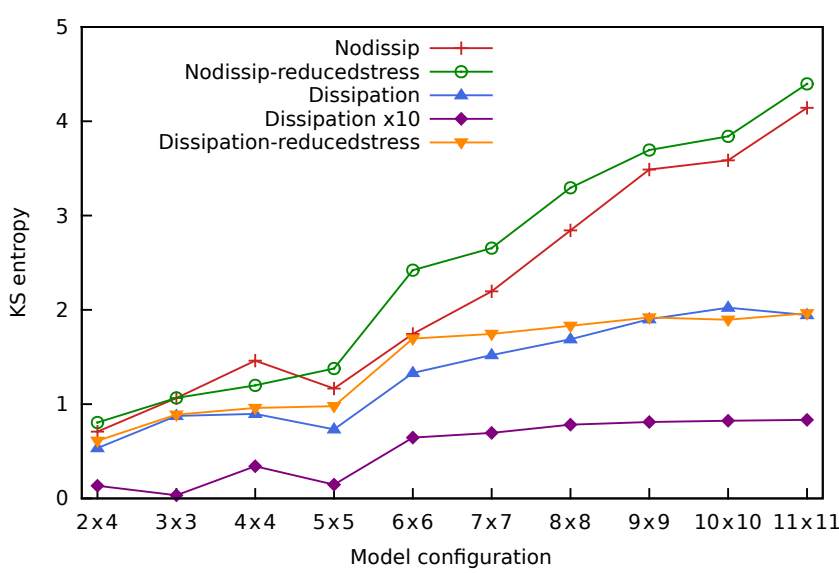

Figure 17. Kolmogorov-Sinai entropy $h_{\mathrm{KS}}$ of MAOOAM as a function of the resolution for the different experiments. Colours as in Fig. 10.

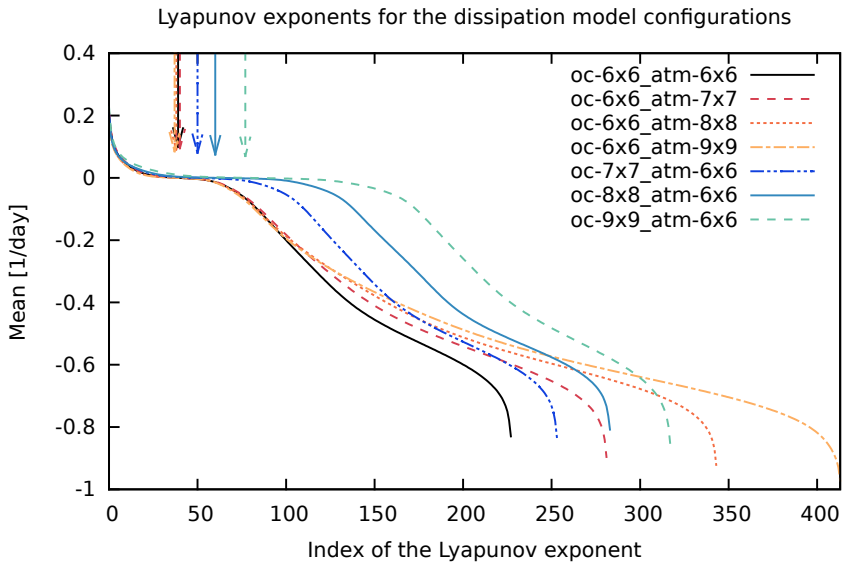

Figure 18. Lyapunov spectra of MAOOAM for the "dissipation" experiment, for different model configurations starting from atm. $6 \times 6$, oc. $6 \times 6$ (black full line). The resolutions of the ocean (dark to light blue lines) and the atmosphere (red to orange lines) are modified separately. Lyapunov exponents are ranked in decreasing order, and the index of the smallest positive Lyapunov exponent is indicated with a downward-pointing arrow for each model configuration.

factory representation of mesoscale and sub-mesoscale processes. PUMA is an atmospheric-only primitive-equation model, which can represent the faster, smaller-scale instabilities associated with processes occurring well below the Rossby deformation radius. On the other side, the lack of an active ocean component removes the presence of very slow scales and does not allow for a built-in scale separation in the dynamics.

We summarise here some findings.

- In PUMA the spectrum of Lyapunov exponents changes in accordance with the paradigm that stronger baroclinic forcing leads to a more unstable atmosphere, as 


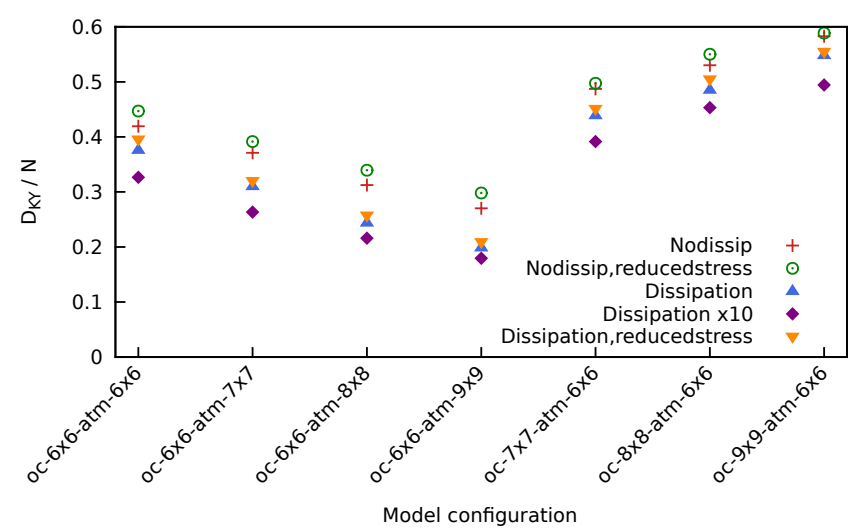

Figure 19. Kaplan-Yorke or Lyapunov dimension $D_{\mathrm{KY}}$ of MAOOAM divided by the total number of dimensions $N$, as a function of the model configuration.

already observed in Schubert and Lucarini (2015) for a quasi-geostrophic model. The model does not feature any separation of scale, as the Lyapunov spectrum is quite smooth. As a result, one cannot clearly distinguish the modes corresponding to baroclinic instability, Kelvin-Helmholtz instability, etc. Despite this, we find that for the lower meridional temperature gradient $\left(\Delta T_{\mathrm{EP}}=50 \mathrm{~K}\right)$ the spectrum features few nearzero negative exponents. Interestingly, this might be related to the presence of blocking, but specific studies with more robust dynamics between blocking and nonblocking situations are necessary to clarify that. Additionally, the results suggest that the FTLEs obey large deviation laws defining the predictability properties at long timescales, including the near-zero exponents. The model can be categorised as being nonuniformly hyperbolic with a trivial central manifold (the direction of the flow).

- For MAOOAM the Lyapunov spectrum is shaped considerably by the presence of the ocean, with a large portion of exponents close to zero. The subspace associated with these exponents corresponds to the central manifold as in the theory of partially hyperbolic systems, and presents features analogous to what was observed in Vannitsem and Lucarini (2016). Furthermore, raising the ocean resolution in MAOOAM clearly increases the number of both positive and negative near-zero Lyapunov exponents, which implies a considerable increase in the Lyapunov dimension of the attractor. Reducing the intensity of the dissipative processes leads, as expected, to an increase in the instability of the model.

One can also conjecture that the set of physical modes, as defined by Yang and Radons (2013), are not yet fully populated since one would expect the isolated modes to be strongly dissipative. This might imply that the resolution necessary to correctly describe the dynamics of
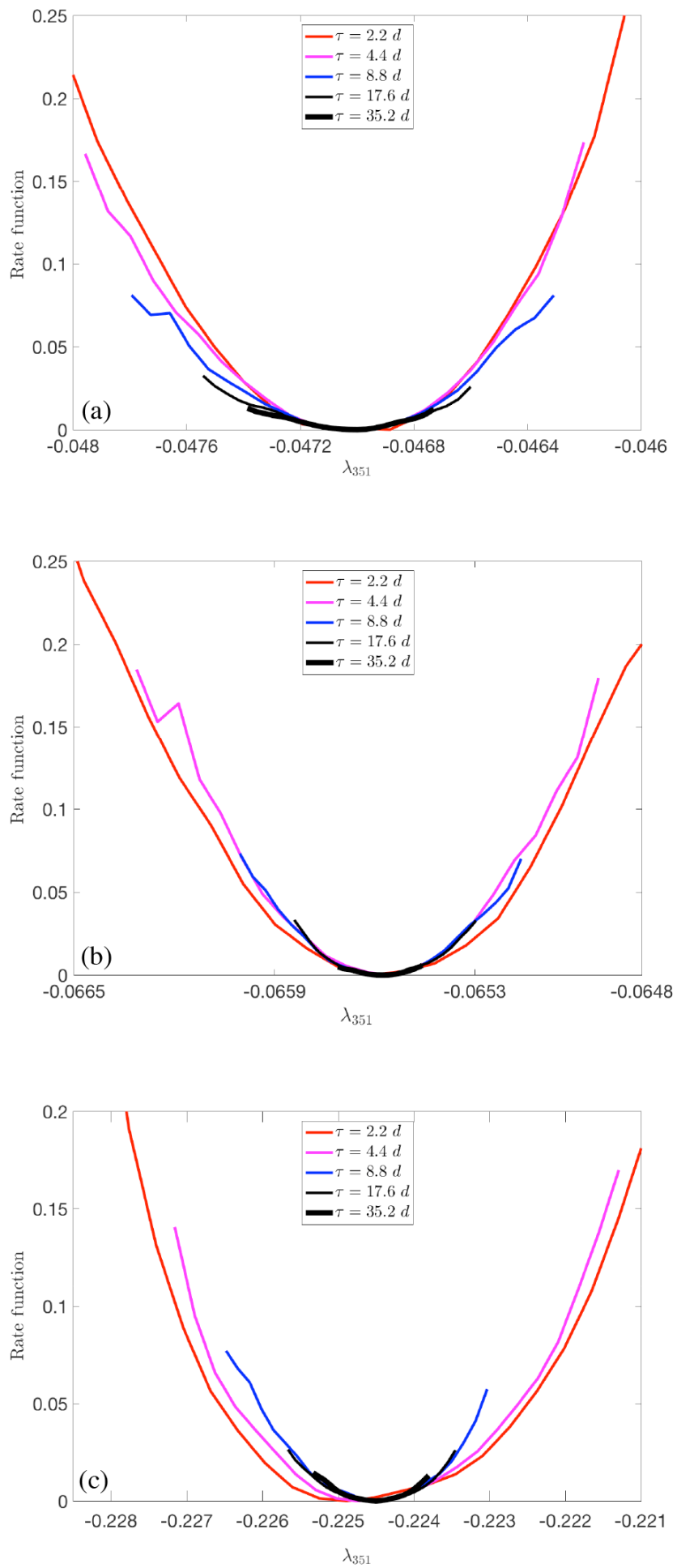

Figure 20. Estimate of the rate function describing the large deviation law of the 351st FTLE for MAOOAM with no dissipation (a), reference value for the dissipation (b), and enhanced dissipation by a factor of 10 (c). 

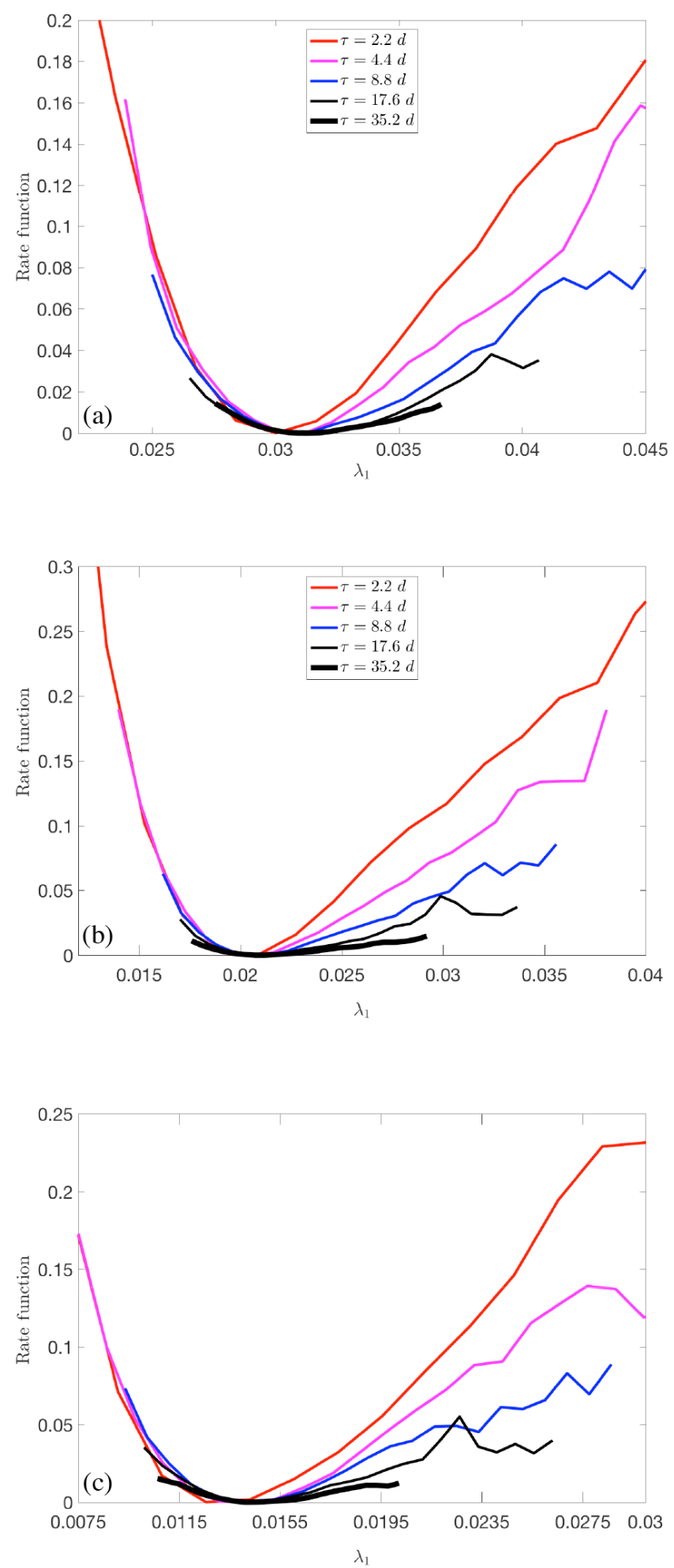

Figure 21. Estimate of the rate function describing the large deviation law of the first FTLE for MAOOAM with no dissipation (a), reference value for the dissipation (b), and enhanced dissipation by a factor of 10 (c). the system is much higher in the ocean. This aspect is well known in ocean dynamics since the unstable baroclinic modes, which play an important role in the ocean variability, can only be resolved with scales smaller than $50 \mathrm{~km}$. Yet the question of defining an appropriate resolution (or more exactly an appropriate set of dynamical modes) for which the dynamics is well captured is still open and the analysis of the CLVs in the spirit of Yang and Radons (2013) and Vannitsem and Lucarini (2016) can help answer this very important question.

The analysis of the FTLEs of MAOOAM reveals some interesting insight into the dynamics. Surprisingly, it is hard to find convergence for the rate functions of the FTLEs, even for those associated with the positive LEs. This may point to the presence of nontrivial ocean influence on the (mostly) atmospheric instabilities.

In the programme we want to develop starting from this investigation, we will employ CLVs in high-dimensional models to tackle various open problems. CLVs allow us to associate growth and decay rates with time-dependent physical modes, and provide a geographical portrait of where instability or damping develops.

First, what is the minimal but sufficient resolution? This is a crucial question, in particular in view of the current computer power needed to perform long-term numerical integrations. A possible way to quantify where this threshold might be, is by means of the different modes identified by Yang and Radons (2013) using CLVs. The CLVs provide information on the optimal splitting of physical modes that effectively describe the dynamics of the system and the highly damped modes. The latter can be considered noisy, purely dissipative terms whose resolution is not necessarily relevant, and are also called isolated modes. Yang and Radons (2013) interpreted them as the result of having a larger number of degrees of freedom in the model than required to resolve all meaningful physical processes. The central feature that allows for the splitting is the angle between the CLVs. If two CLVs display angles around $90^{\circ}$ and bounded away from $0^{\circ}$, these directions in phase space can be naturally split. Being able to describe the physical modes is deemed essential for satisfactorily reproducing the so-called inertial manifold. The inertial manifold contains the effective finite-dimensional dynamics of the system, which, we recall, is originally infinitedimensional if we represent a continuum system described by (S)PDEs. In particular, it would be interesting to determine how the threshold for resolving the inertial manifold varies in a purely atmospheric model compared to a coupled atmosphere-ocean model. Additionally, the analysis of geometry of the tangent space can clarify to what extent a system can be treated - effectively, not rigorously - as hyperbolic versus partially hyperbolic. As explained in Vannitsem and Lucarini (2016), this has profound implications for predictability. 
Second, we want to understand multiscale instabilities better and find out what the driving processes are behind their growth. Here, the covariance of the CLVs with the tangent linear equation is the key for understanding instabilities and their properties far away from an equilibrium. Traditionally, even in a chaotic setting such an analysis relied on classic normal mode instability of fixed stationary states (e.g. Charney, 1947; Eady, 1949; Pedlosky, 1964) to explain phenomena like the baroclinic and barotropic instability. This approach has been very beneficial but has many known shortcomings for the understanding of highly nonlinear phenomena such as wave-wave interactions (Speranza and Malguzzi, 1988) or regime switching like blocking (Pelly and Hoskins, 2003). Additionally, CLVs will allow us to better understand coupled ocean-atmospheric modes. We wish to develop our future programme in line with Schubert and Lucarini $(2015,2016)$, who demonstrated that CLVs give a picture of which types of instabilities exist in an atmospheric quasi-geostrophic (QG) two-layer model and of the energetics behind them. For example, the fastest modes can be almost exclusively barotropically unstable even though traditional normal mode analysis suggests the most unstable modes are driven by baroclinic energy conversion. Given these findings, we expect an even more diverse mixture of different types of instabilities in multiscale systems such as PUMA or MAOOAM. This approach is a promising alternative to restricting the analysis to either studying idealised life cycles of instabilities (Plougonven and Zhang, 2014) or studying yet again normal modes (Molemaker et al., 2005).

Code availability. The PUMA model is a part of PLASIM, for which the source code can be downloaded at https://www.mi.uni-hamburg.de/en/arbeitsgruppen/ theoretische-meteorologie/modelle/sources/plasim.tgz (last access: 14 March 2018). The source code for the latest version of MAOOAM is available at http://github.com/Climdyn/MAOOAM (last access: 13 December 2017). The version of MAOOAM that was used to compute the Lyapunov exponents is archived at https://doi.org/10.5281/zenodo.1198650 (De Cruz et al., 2018).

Data availability. The Lyapunov spectra of the different PUMA and MAOOAM configurations, which were computed using the Benettin algorithm, are available as a Supplement. 


\section{Appendix A: Study of the convergence of the rate function}

We have performed an additional analysis that focuses on the convergence of the standard deviation $\sigma$ of the FTLE as a function of the length $t_{\text {ave }}$ used to compute the block averages. Indeed, $\sigma$ is expected to scale according to $t_{\text {ave }}^{-\frac{1}{2}}$. Furthermore, one expects the value of $\sigma^{2} \cdot t_{\text {ave }}$ to level off at the value of the diffusion coefficient $D$ if there is convergence to the central limit theorem. The diffusion coefficient $D$ is the inverse of the second derivative of the rate function at its minimum; see e.g. Kuptsov and Politi (2011).

\section{A1 PUMA}

The scaling of $\sigma$ versus $t_{\text {ave }}$ has the expected behaviour for both experiments conducted with PUMA, as shown in Fig. A1. This is also apparent in the convergence of $\sigma^{2} \cdot t_{\text {ave }}$, shown in Fig. A2. While the value of $D$ fluctuates, it has the right order of magnitude.

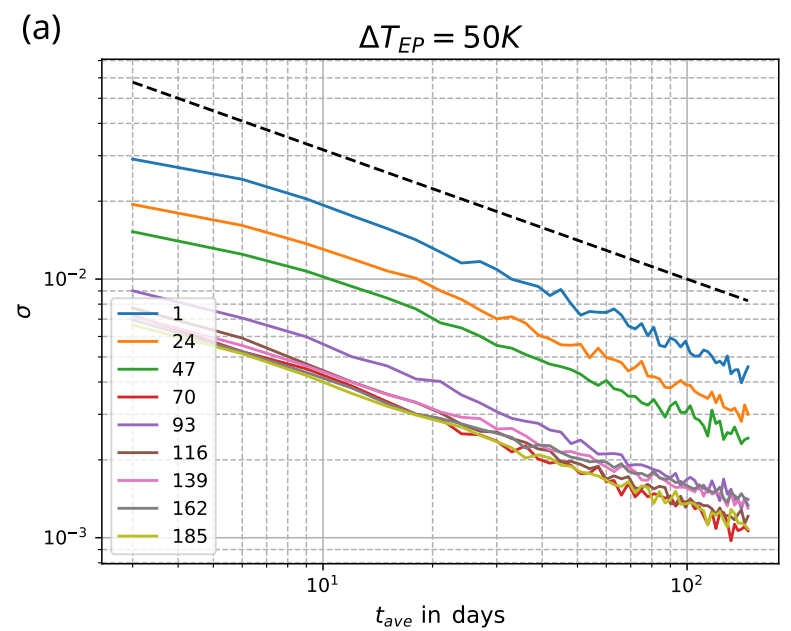

\section{A2 MAOOAM}

The decorrelation time of the near-zero FTLEs in MAOOAM is extremely long. Accordingly, the time intervals $t_{\mathrm{ave}}$, used to determine the rate functions associated with the FTLE, are expected to be insufficient to robustly define large deviation laws describing the statistics of the FTLEs. This is confirmed by the behaviour of $\sigma$ versus $t_{\text {ave }}$ for the " $9 \times 9$ " model configuration, shown in Fig. A3. A discrepancy between $D$ and $\sigma^{2} \cdot t_{\text {ave }}$ is apparent for LE 100 in all experiments shown in Fig. A4. Even for positive or strongly negative LEs, we are not yet in the regime of convergence to the central limit theorem. Note however that an integration time of 614 years was used to compute the Lyapunov spectra, longer than the time series used in this analysis.

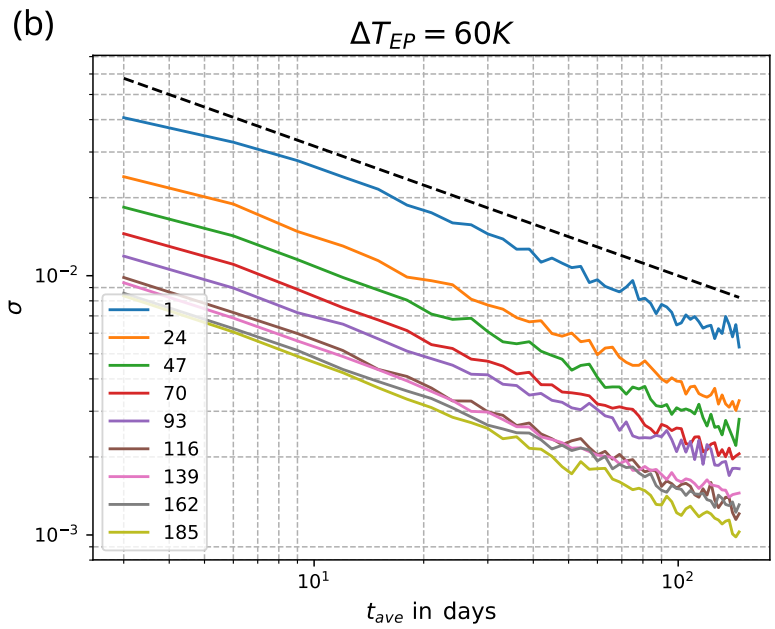

Figure A1. Standard deviation $\sigma$ as a function of the block averaging length $t_{\text {ave }}$ for different Lyapunov exponents of the PUMA model. The Lyapunov index is shown in the legend. Panel (a) shows the results for a temperature gradient $\Delta T_{\mathrm{EP}}$ of $50 \mathrm{~K}$, panel (b) for $60 \mathrm{~K}$. The black dashed line corresponds to $t_{\text {ave }}^{-\frac{1}{2}}$ scaling. 

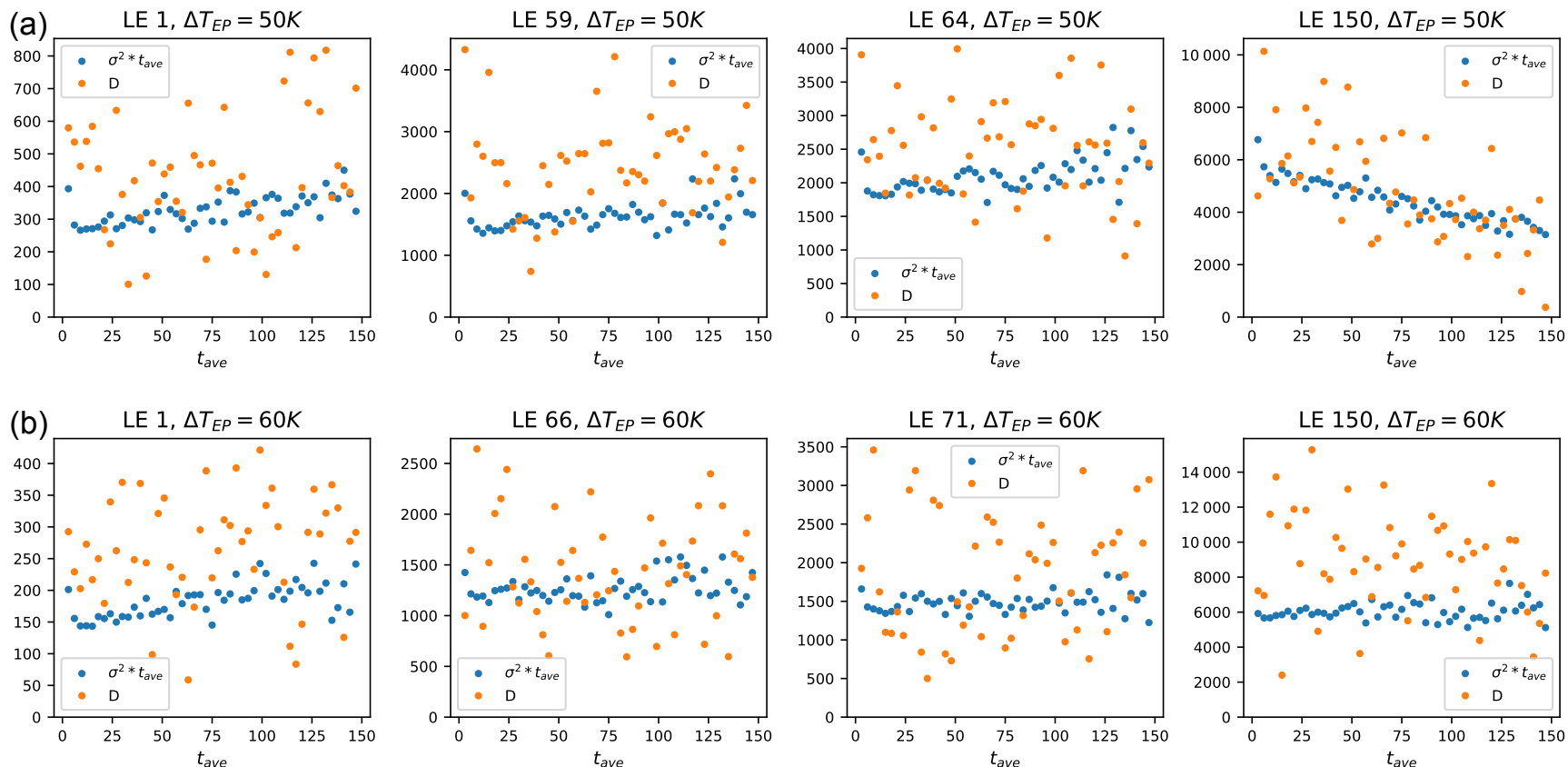

Figure A2. The metric $\sigma^{2} \cdot t_{\text {ave }}$ versus the diffusion coefficient $D$, derived from the inverse of the second derivative at the minimum of the rate function, as a function of the block averaging length $t_{\text {ave }}$ for different Lyapunov exponents of the PUMA model. The Lyapunov index is shown in the title. Panels (a) show the results for a temperature gradient of $50 \mathrm{~K}$, panels (b) for $60 \mathrm{~K}$. 

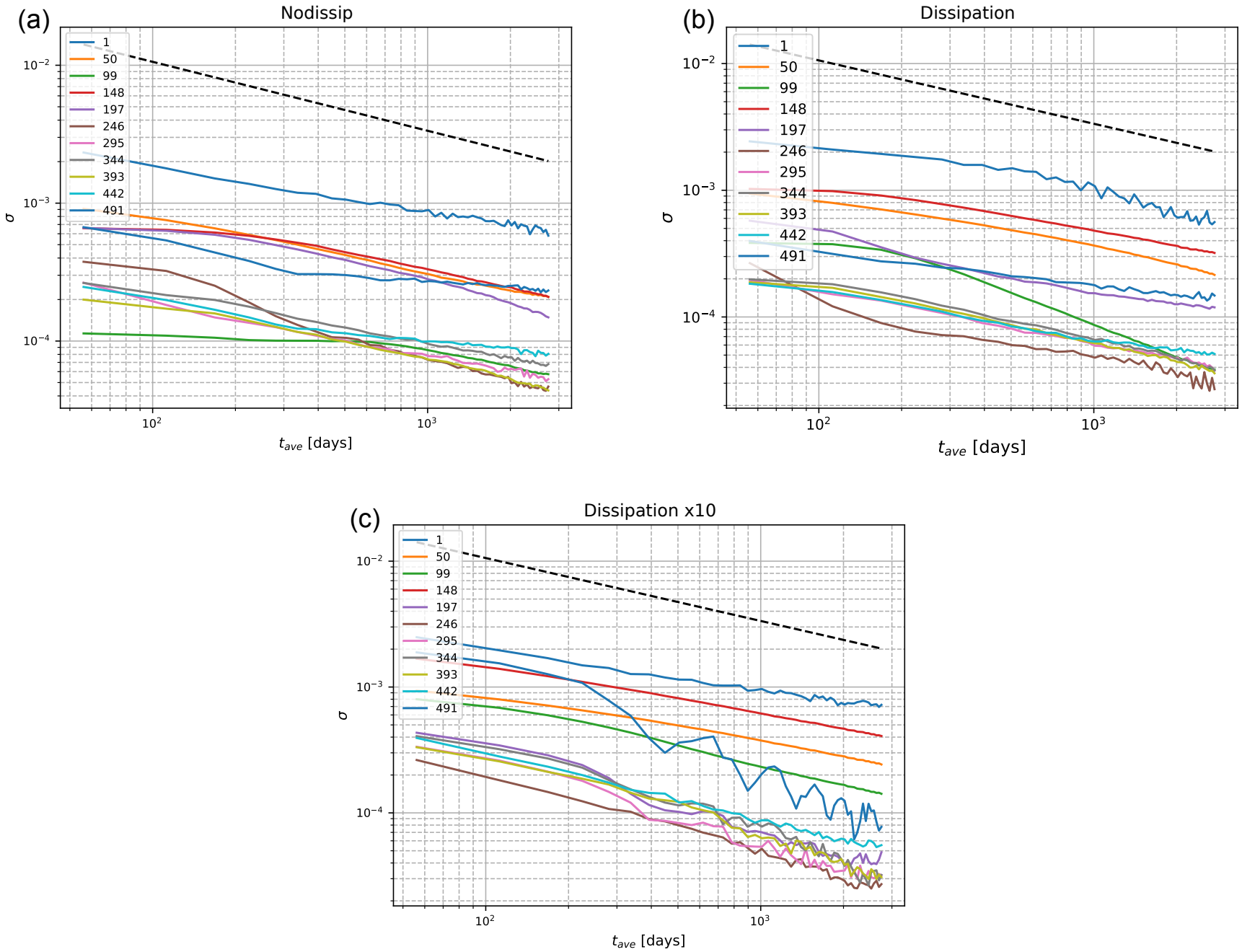

Figure A3. Standard deviation $\sigma$ as a function of the block averaging length $t_{\text {ave }}$ for different Lyapunov exponents of the " $9 \times 9$ " configuration of MAOOAM. The Lyapunov index is shown in the legend. Panel (a) shows the results for the experiment without scale-dependent dissipation, panel (b) corresponds to the reference value for dissipation, and panel (c) shows the enhanced dissipation results. The black dashed line corresponds to $t_{\text {ave }}^{-\frac{1}{2}}$ scaling. 
(a)

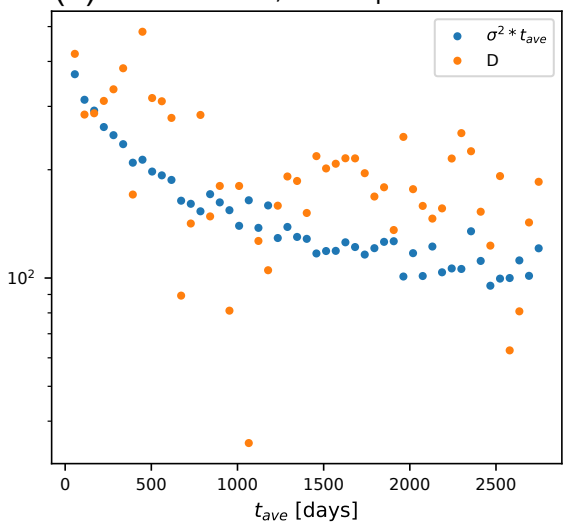

(b)

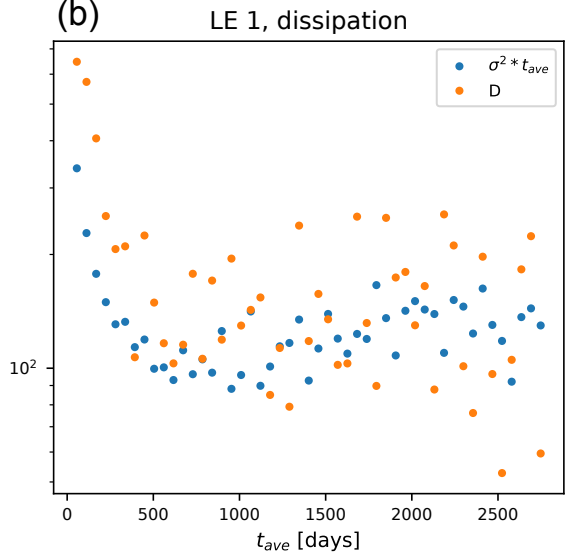

(c)

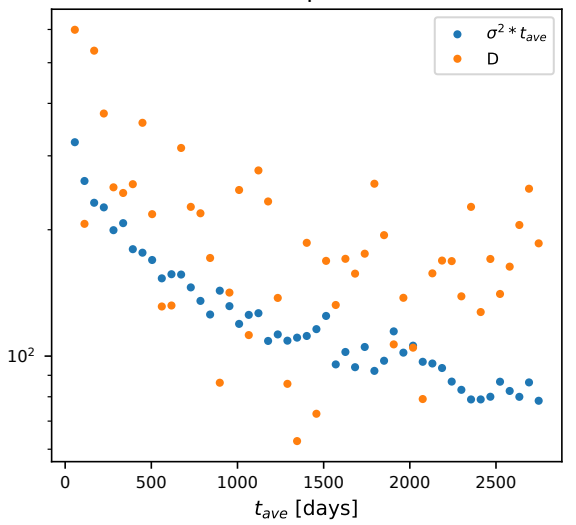

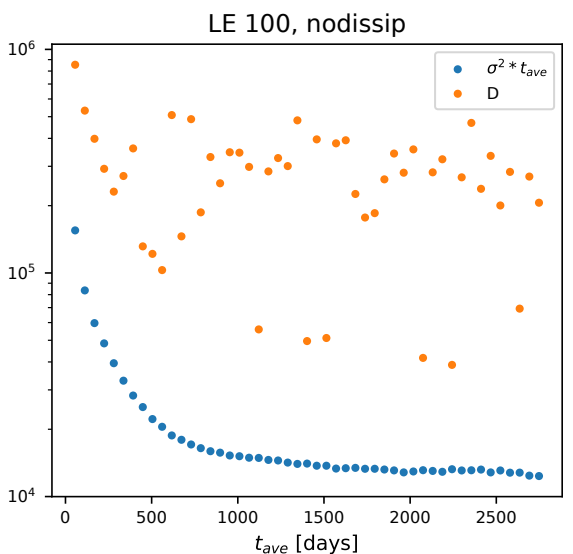
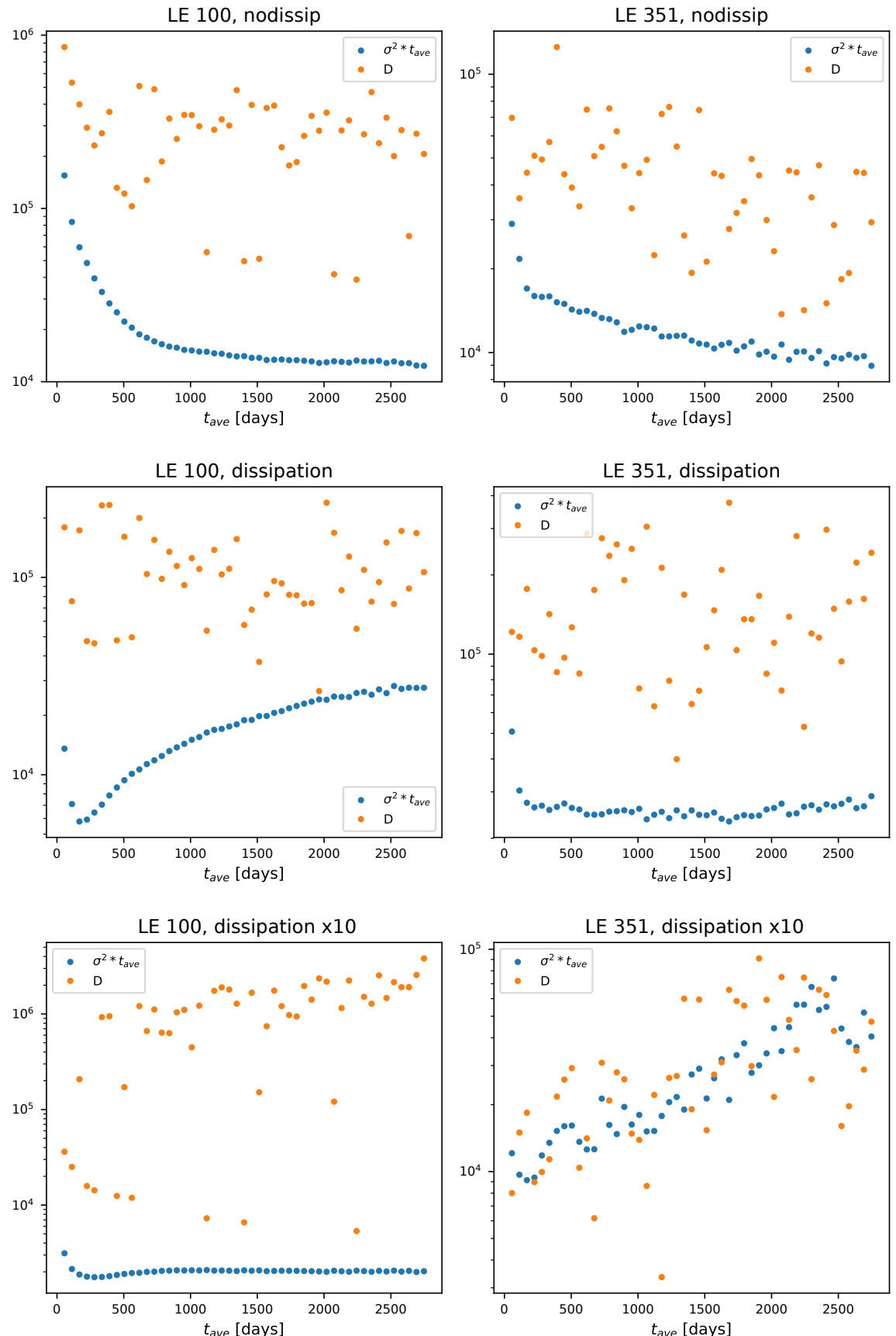

Figure A4. The metric $\sigma^{2} \cdot t_{\text {ave }}$ versus the diffusion coefficient $D$, derived from the inverse of the second derivative at the minimum of the rate function, as a function of the block averaging length $t_{\text {ave }}$ for different Lyapunov exponents of the " $9 \times 9$ " configuration of MAOOAM. Note the logarithmic scale of the $y$-axis, in contrast to Fig. A2. The Lyapunov index is shown in the title. Panels (a) show the results for the experiment without scale-dependent dissipation, panels (b) correspond to the reference value for dissipation, and panels (c) show the results for an enhanced dissipation coefficient. 
Supplement. The supplement related to this article is available online at: https://doi.org/10.5194/npg-25-387-2018-supplement.

Author contributions. VL and SS performed the analysis of PUMA. SS wrote the code to compute the LEs for PUMA. LDC and SV performed the analysis of MAOOAM. JD and SS wrote the code to compute the LEs for MAOOAM. LDC, SV and JD wrote MAOOAM. VL analysed the FTLEs in MAOOAM. All authors contributed to the writing of the manuscript.

Competing interests. Stéphane Vannitsem and Valerio Lucarini are members of the editorial board of the journal. The other authors declare that they have no conflict of interest.

Special issue statement. This article is part of the special issue "Numerical modeling, predictability and data assimilation in weather, ocean and climate: A special issue honoring the legacy of Anna Trevisan (1946-2016)". It is not associated with a conference.

Acknowledgements. The authors would like to thank Stephen G. Penny and an anonymous reviewer for improving the manuscript with their constructive comments.

Lesley De Cruz was supported by the BELSPO under contract BR/165/A2/Mass2Ant. Jonathan Demaeyer was supported by BELSPO under contract BR/121/A2/STOCHCLIM. Valerio Lucarini and Sebastian Schubert were supported by the DFG through the contract SFB/Transregio TRR181.

Edited by: Juan Manuel Lopez

Reviewed by: Stephen G. Penny and one anonymous referee

\section{References}

Abarbanel, H. D., Brown, R., and Kennel, M. B.: Variation of Lyapunov exponents on a strange attractor, J. Nonlinear Sci., 1, 175199, https://doi.org/10.1007/BF01209065, 1991.

Barsugli, J. J. and Battisti, D. S.: The Basic Effects of Atmosphere-Ocean Thermal Coupling on Midlatitude Variability, J. Atmos. Sci., 55, 477-493, https://doi.org/10.1175/15200469(1998)055<0477:TBEOAO>2.0.CO;2, 1998.

Benettin, G., Galgani, L., Giorgilli, A., and Strelcyn, J.-M.: Lyapunov Characteristic Exponents for smooth dynamical systems and for hamiltonian systems; a method for computing all of them. Part 1: Theory, Meccanica, 15, 9-20, https://doi.org/10.1007/BF02128236, 1980.

Boffetta, G., Cencini, M., Falcioni, M., and Vulpiani, A.: Predictability: a way to characterize complexity, Phys. Rep., 356, 367-474, https://doi.org/10.1016/S0370-1573(01)00025-4, 2002.

Boschi, R., Lucarini, V., and Pascale, S.: Bistability of the climate around the habitable zone: A thermodynamic investigation, Icarus, 226, 1724-1742, https://doi.org/10.1016/j.icarus.2013.03.017, 2013.
Carrassi, A., Trevisan, A., Descamps, L., Talagrand, O., and Uboldi, F.: Controlling instabilities along a 3DVar analysis cycle by assimilating in the unstable subspace: a comparison with the EnKF, Nonlin. Processes Geophys., 15, 503-521, https://doi.org/10.5194/npg-15-503-2008, 2008.

Cencini, M. and Ginelli, F.: Lyapunov analysis: from dynamical systems theory to applications, J. Phys. A-Math. Theor., 46, 250301, https://doi.org/10.1088/1751-8113/46/25/250301, 2013.

Cencini, M., Cecconi, F., and Vulpiani, A.: Chaos: From Simple Models to Complex Systems, vol. 17 of Series on Advances in Statistical Mechanics, World Scientific, https://doi.org/10.1142/7351, 2010.

Charney, J. G.: The Dynamics Of Long Waves In A Baroclinic Current, J. Meteorol., 4, 136-162, https://doi.org/10.1175/15200469(1947)004<0136:TDOLWI>2.0.CO;2, 1947.

Charney, J. G. and Straus, D. M.: Form-Drag Instability, Multiple Equilibria and Propagating Planetary Waves in Baroclinic, Orographically Forced, Planetary Wave Systems, J. Atmos. Sci., 37, 1157-1176, https://doi.org/10.1175/15200469(1980)037<1157:FDIMEA>2.0.CO;2, 1980.

De Cruz, L., Demaeyer, J., and Vannitsem, S.: The Modular Arbitrary-Order Ocean-Atmosphere Model: MAOOAM v1.0, Geosci. Model Dev., 9, 2793-2808, https://doi.org/10.5194/gmd9-2793-2016, 2016.

De Cruz, L., Demaeyer, J., Schubert, S., and Vannitsem, S.: MAOOAM v1.3.1-lyapunov, Zenodo, https://doi.org/10.5281/zenodo.1198650, 2018.

Demaeyer, J. and Vannitsem, S.: Stochastic parametrization of subgrid-scale processes in coupled ocean-atmosphere systems: benefits and limitations of response theory, Q. J. Roy. Meteor. Soc., 143, 881-896, https://doi.org/10.1002/qj.2973, 2016.

Eady, E. T.: Long Waves and Cyclone Waves, Tellus, 1, 33-52, https://doi.org/10.1111/j.2153-3490.1949.tb01265.x, 1949.

Eckmann, J. and Ruelle, D.: Ergodic theory of chaos and strange attractors, Rev. Mod. Phys., 57, 617-656, https://doi.org/10.1103/RevModPhys.57.617, 1985.

Ershov, S. V. and Potapov, A. B.: On the concept of stationary Lyapunov basis, Physica D, 118, 167-198, https://doi.org/10.1016/S0167-2789(98)00013-X, 1998.

Fraedrich, K. and Kirk, E.: The portable university model of the atmosphere (PUMA): Storm track dynamics and low-frequency variability, Meteorol. Z., 14, 735-745, https://doi.org/10.1127/0941-2948/2005/0074, 2005.

Fraedrich, K., Kirk, E., and Lunkeit, F.: Portable University Model of the Atmosphere, Tech. rep., DKRZ, Hamburg, available at: https://www.dkrz.de/mms//pdf/reports/ReportNo.16.pdf (last access: 17 November 2017), 1998.

Frederickson, P., Kaplan, J. L., Yorke, E. D., and Yorke, J. A.: The Liapunov dimension of strange attractors, J. Differ. Equations, 49, 185-207, https://doi.org/10.1016/00220396(83)90011-6, 1983.

Fujisaka, H.: Statistical dynamics generated by fluctuations of local Lyapunov exponents, Prog. Theor. Phys., 70, 1264-1275, https://doi.org/10.1143/PTP.70.1264, 1983.

Gallavotti, G. and Cohen, E. G. D.: Dynamical Ensembles in Nonequilibrium Statistical Mechanics, Phys. Rev. Lett., 74, 2694-2697, https://doi.org/10.1103/PhysRevLett.74.2694, 1995.

Gallavotti, G.: Nonequilibrium and Irreversibility, Springer, New York, USA, 2014. 
Gallavotti, G. and Lucarini, V.: Equivalence of Non-equilibrium Ensembles and Representation of Friction in Turbulent Flows: The Lorenz 96 Model, J. Stat. Phys., 156, 1027-1065, https://doi.org/10.1007/s10955-014-1051-6, 2014.

Gallez, D. and Babloyantz, A.: Lyapunov exponents for nonuniform attractors, Phys. Lett., 161, 247-254, https://doi.org/10.1016/0375-9601(91)90012-W, 1991.

Giering, R. and Kaminski, T.: Applying TAF to generate efficient derivative code of Fortran 77-95 programs, PAMM, 2, 54-57, https://doi.org/10.1002/pamm.200310014, 2003.

Ginelli, F., Poggi, P., Turchi, A., Chaté, H., Livi, R., and Politi, A.: Characterizing dynamics with covariant Lyapunov vectors, Phys. Rev. Lett., 99, 130601, https://doi.org/10.1103/PhysRevLett.99.130601, 2007.

Held, I. M. and Suarez, M. J.: A Proposal for the Intercomparison of the Dynamical Cores of Atmospheric General Circulation Models, B. Am. Meteorol. Soc., 75, 1825-1830, https://doi.org/10.1175/15200477(1994)075<1825:APFTIO>2.0.CO;2, 1994.

Holden, P. B., Edwards, N. R., Garthwaite, P. H., Fraedrich, K., Lunkeit, F., Kirk, E., Labriet, M., Kanudia, A., and Babonneau, F.: PLASIM-ENTSem v1.0: a spatio-temporal emulator of future climate change for impacts assessment, Geosci. Model Dev., 7, 433-451, https://doi.org/10.5194/gmd-7-433-2014, 2014.

Holton, J. R.: An Introduction To Dynamic Meteorology, 4th edn., Academic Press, San Diego, California, USA, 2004.

Kalnay, E.: Atmospheric Modeling, Data Assimilation, and Predictability, Cambridge University Press, New York, USA, 2003.

Kaplan, J. L. and Yorke, J. A.: Chaotic behavior of multidimensional difference equations, in: Functional Differential Equations and Approximation of Fixed Points: Proceedings, Bonn, July 1978, edited by: Peitgen, H.-O. and Walther, H.-O., SpringerVerlag, Berlin, Germany, 204-227, 1979.

Kifer, Y.: Large deviations in dynamical systems and stochastic processes, T. Am. Math. Soc., 321, 505-524, https://doi.org/10.1090/S0002-9947-1990-1025756-7, 1990.

Klein, R.: Scale-Dependent Models for Atmospheric Flows, Annu. Rev. Fluid Mech., 42, 249-274, https://doi.org/10.1146/annurevfluid-121108-145537, 2010.

Kuptsov, P. V. and Politi, A.: Large-deviation approach to space-time chaos, Phys. Rev. Lett., 107, 114101, https://doi.org/10.1103/PhysRevLett.107.114101, 2011.

Laffargue, T., Lam, K.-D. N. T., Kurchan, J., and Tailleur, J.: Large deviations of Lyapunov exponents, J. Phys. A-Math. Theor., 46, 254002, https://doi.org/10.1088/1751-8113/46/25/254002, 2013.

Legras, B. and Ghil, M.: Persistent Anomalies, Blocking and Variations in Atmospheric Predictability, J. Atmos. Sci., 42, 433-471, https://doi.org/10.1175/15200469(1985)042<0433:PABAVI>2.0.CO;2, 1985.

Legras, B. and Vautard, R.: A Guide to Liapunov vectors, in: Proceedings 1995 ECMWF Seminar on Predictability, 4-8 September 1995, Reading, UK, vol. 1, 143-156, available at: http: //gershwin.ens.fr/legras/publis/liapunov.pdf (last access: 13 December 2017), 1995.

Lesieur, M.: Introduction to Turbulence in Fluid Mechanics, in: Turbulence in Fluids: Stochastic and Numerical Modelling, Springer, Dordrecht, the Netherlands, 1-18, https://doi.org/10.1007/978-94-009-0533-7_1, 1990.
Lorenz, E. N.: Deterministic Nonperiodic Flow, J. Atmos. Sci., 20, 130-141, https://doi.org/10.1175/15200469(1963)020<0130:DNF>2.0.CO;2, 1963.

Lucarini, V. and Fraedrich, K.: Symmetry breaking, mixing, instability, and low-frequency variability in a minimal Lorenz-like system, Phys. Rev. E, 80, 026313, https://doi.org/10.1103/PhysRevE.80.026313, 2009.

Lucarini, V. and Ragone, F.: Energetics Of Climate Models: Net Energy Balance And Meridional Enthalpy Transport, Rev. Geophys., 49, RG1001, https://doi.org/10.1029/2009RG000323, 2011.

Lucarini, V., Speranza, A., and Vitolo, R.: Parametric smoothness and self-scaling of the statistical properties of a minimal climate model: What beyond the mean field theories?, Physica D, 234, 105-123, https://doi.org/10.1016/j.physd.2007.07.006, 2007.

Lucarini, V., Fraedrich, K., and Lunkeit, F.: Thermodynamic analysis of snowball earth hysteresis experiment: efficiency, entropy production and irreversibility, Q. J. Roy. Meteor. Soc., 136,2-11, https://doi.org/10.1002/qj.543, 2010.

Lucarini, V., Blender, R., Herbert, C., Ragone, F., Pascale, S., and Wouters, J.: Mathematical and physical ideas for climate science, Rev. Geophys., 52, 809-859, https://doi.org/10.1002/2013RG000446, 2014.

Lucarini, V., Ragone, F., and Lunkeit, F.: Predicting climate change using response theory: global averages and spatial patterns, J. Stat. Phys., 166, 1036-1064, https://doi.org/10.1007/s10955016-1506-z, 2017.

Manneville, P.: Liapounov exponents for the Kuramoto-Sivashinsky model, in: Macroscopic Modelling of Turbulent Flows, Springer Berlin Heidelberg, Berlin, Heidelberg, Germany, vol. 230, 319326, https://doi.org/10.1007/3-540-15644-5_26, 1985.

Manneville, P.: Dissipative structures and weak turbulence, in: Chaos - The Interplay Between Stochastic and Deterministic Behaviour, Springer Berlin Heidelberg, Berlin, Heidelberg, Germany, vol. 457, 257-272, https://doi.org/10.1007/3-540-601880_59, 1995.

Molemaker, M. J., McWilliams, J. C., and Yavneh, I.: Baroclinic Instability and Loss of Balance, J. Phys. Oceanogr., 35, 15051517, https://doi.org/10.1175/JPO2770.1, 2005.

Nese, J. M. and Dutton, J. A.: Quantifying predictability variations in a low-order occan-atmosphere model: a dynamical systems approach, J. Climate, 6, 185-204, https://doi.org/10.1175/15200442(1993)006<0185:QPVIAL>2.0.CO;2, 1993.

Nicolis, C.: Dynamics of model error: The role of the boundary conditions, J. Atmos. Sci., 64, 204-215, https://doi.org/10.1175/JAS3806.1, 2007.

Nicolis, C., Nicolis, G., and Wang, Q.: Sensitivity To Initial Conditions In Spatially Distributed Systems, Int. J. Bifurcat. Chaos, 02, 263-269, https://doi.org/10.1142/S0218127492000276, 1992.

Nicolis, C., Perdigao, R. A., and Vannitsem, S.: Dynamics of prediction errors under the combined effect of initial condition and model errors, J. Atmos. Sci., 66, 766-778, https://doi.org/10.1175/2008JAS2781.1, 2009.

Oseledets, V.: Oseledets theorem, Scholarpedia, 3, 1846, https://doi.org/10.4249/scholarpedia.1846, 2008.

Oseledets, V. I.: A multiplicative ergodic theorem. Characteristic Ljapunov, exponents of dynamical systems, Transactions of the Moscow Mathematical Society, 19, 179-210, 1968. 
Ott, E.: Chaos in Dynamical Systems - 2nd Edition, Cambridge University Press, https://doi.org/10.1017/CBO9780511803260, 2002.

Pazó, D., Szendro, I. G., López, J. M., and Rodríguez, M. A.: Structure of characteristic Lyapunov vectors in spatiotemporal chaos, Phys. Rev. E, 78, 1-9, https://doi.org/10.1103/PhysRevE.78.016209, 2008.

Pazó, D., Rodríguez, M. A., and López, J. M.: Spatiotemporal evolution of perturbations in ensembles initialized by bred, Lyapunov and singular vectors, Tellus A, 62, 10-23, https://doi.org/10.1111/j.1600-0870.2009.00419.x, 2010.

Pazó, D., López, J. M., and Politi, A.: Universal scaling of Lyapunov-exponent fluctuations in space-time chaos, Phys. Rev. E, 87, 062909, https://doi.org/10.1103/PhysRevE.87.062909, 2013.

Pedlosky, J.: The Stability of Currents in the Atmosphere and the Ocean: Part II, J. Atmos. Sci., 21, 201-219, https://doi.org/10.1175/15200469(1964)021<0342:TSOCIT>2.0.CO;2, 1964.

Pelly, J. L. and Hoskins, B. J.: A New Perspective on Blocking, J. Atmos. Sci., 60, 743-755, https://doi.org/10.1175/15200469(2003)060<0743:ANPOB>2.0.CO;2, 2003.

Pesin, Y.: Lectures on Partial Hyperbolicity and Stable Ergodicity, European Mathematical Society, Zurich, Switzerland, 2004.

Pierini, S.: Low-frequency variability, coherence resonance, and phase selection in a low-order model of the winddriven ocean circulation, J. Phys. Oceanogr., 41, 1585-1604, https://doi.org/10.1175/JPO-D-10-05018.1, 2011.

Plougonven, R. and Zhang, F.: Internal gravity waves from atmospheric jets and fronts, Rev. Geophys., 52, 33-76, https://doi.org/10.1002/2012RG000419, 2014.

Ragone, F., Lucarini, V., and Lunkeit, F.: A new framework for climate sensitivity and prediction: a modelling perspective, Clim. Dynam., 46, 1459-1471, https://doi.org/10.1007/s00382015-2657-3, 2016.

Ruelle, D.: Ergodic theory of differentiable dynamical systems, Publ. Math., 50, 27-58, https://doi.org/10.1007/BF02684768, 1979.

Ruelle, D.: A review of linear response theory for general differentiable dynamical systems, Nonlinearity, 22, 855-870, https://doi.org/10.1088/0951-7715/22/4/009, 2009.

Schalge, B., Blender, R., Wouters, J., Fraedrich, K., and Lunkeit, F.: Evidence for a fluctuation theorem in an atmospheric circulation model, Phys. Rev. E, 87, 8760, https://doi.org/10.1103/PhysRevE.87.052113, 2013.

Schneider, T.: The General Circulation of the Atmosphere, Annu. Rev. Earth Pl. Sc., 34, 655-688, https://doi.org/10.1146/annurev.earth.34.031405.125144, 2006.

Schubert, S. and Lucarini, V.: Covariant Lyapunov vectors of a quasi-geostrophic baroclinic model: Analysis of instabilities and feedbacks, Q. J. Roy. Meteor. Soc., 141, 3040-3055, https://doi.org/10.1002/qj.2588, 2015.

Schubert, S. and Lucarini, V.: Dynamical analysis of blocking events: spatial and temporal fluctuations of covariant Lyapunov vectors, Q. J. Roy. Meteor. Soc., 142, 2143-2158, https://doi.org/10.1002/qj.2808, 2016.

Scott, D. W.: On optimal and data-based histograms, Biometrika, 66, 605-610, 1979.
Shimada, I. and Nagashima, T.: A Numerical Approach to Ergodic Problem of Dissipative Dynamical Systems, Prog. Theor. Phys., 61, 1605-1616, https://doi.org/10.1143/PTP.61.1605, 1979.

Speranza, A. and Malguzzi, P.: The Statistical Properties of a Zonal Jet in a Baroclinic Atmosphere: A Semilinear Approach. Part I: Quasi-geostrophic, Two-Layer Model Atmosphere, J. Atmos. Sci., 45, 3046-3062, https://doi.org/10.1175/15200469(1988)045<3046:TSPOAZ>2.0.CO;2, 1988.

Sprott, J. C.: Elegant Chaos, World Scientific, https://doi.org/10.1142/7183, 2010.

Thompson, P. D.: Uncertainty of initial state as a factor in the predictability of large scale atmospheric flow patterns, Tellus, 9, 275-295, https://doi.org/10.3402/tellusa.v9i3.9111, 1957.

Tibaldi, S. and Molteni, F.: On the operational predictability of blocking, Tellus A, 42, 343-365, https://doi.org/10.1034/j.16000870.1990.t01-2-00003.x, 1990.

Touchette, H.: The large deviation approach to statistical mechanics, Phys. Rep., 478, 1-69, https://doi.org/10.1016/j.physrep.2009.05.002, 2009.

Trevisan, A. and Pancotti, F.: Periodic Orbits, Lyapunov Vectors, and Singular Vectors in the Lorenz System, J. Atmos. Sci., 55, 390-398, https://doi.org/10.1175/15200469(1998)055<0390:POLVAS>2.0.CO;2, 1998.

Trevisan, A., D'Isidoro, M., and Talagrand, O.: Four-dimensional variational assimilation in the unstable subspace and the optimal subspace dimension, Q. J. Roy. Meteor. Soc., 136, 487-496, 2010.

Vallis, G. K.: Atmospheric and oceanic fluid dynamics: fundamentals and large-scale circulation, Cambridge University Press, New York, USA, 2006.

Van der Avoird, E., Dijkstra, H., Nauw, J., and Schuurmans, C.: Nonlinearly induced low-frequency variability in a midlatitude coupled ocean-atmosphere model of intermediate complexity, Clim. Dynam., 19, 303-320, https://doi.org/10.1007/s00382001-0220-x, 2002.

Vannitsem, S.: Predictability of large-scale atmospheric motions: Lyapunov exponents and error dynamics, Chaos, 27, 32101, https://doi.org/10.1063/1.4979042, 2017.

Vannitsem, S. and De Cruz, L.: A 24-variable low-order coupled ocean-atmosphere model: OA-QG-WS v2, Geosci. Model Dev., 7, 649-662, https://doi.org/10.5194/gmd-7-649-2014, 2014.

Vannitsem, S. and Lucarini, V.: Statistical and Dynamical Properties of Covariant Lyapunov Vectors in a Coupled AtmosphereOcean Model - Multiscale Effects, Geometric Degeneracy, and Error Dynamics, J. Phys. A-Math. Theor., 49, 224001, https://doi.org/10.1088/1751-8113/49/22/224001, 2016.

Vannitsem, S. and Nicolis, C.: Predictability experiments on a simplified thermal convection model: The role of spatial scales, J. Geophys. Res., 99, 10377, https://doi.org/10.1029/94JD00248, 1994.

Vannitsem, S. and Nicolis, C.: Error Growth Dynamics In Spatially Extended Systems, Int. J. Bifurcat. Chaos, 6, 2223-2235, https://doi.org/10.1142/S0218127496001466, 1996.

Vannitsem, S. and Nicolis, C.: Lyapunov Vectors and Error Growth Patterns in a T21L3 Quasigeostrophic Model, J. Atmos. Sci., 54, 347-361, https://doi.org/10.1175/15200469(1997)054<0347:LVAEGP>2.0.CO;2, 1997.

Vannitsem, S., Demaeyer, J., De Cruz, L., and Ghil, M.: Lowfrequency variability and heat transport in a low-order nonlin- 
ear coupled ocean-atmosphere model, Physica D, 309, 71-85, https://doi.org/10.1016/j.physd.2015.07.006, 2015.

Wolfe, C. L. and Samelson, R. M.: An efficient method for recovering Lyapunov vectors from singular vectors, Tellus A, 59, 355366, https://doi.org/10.1111/j.1600-0870.2007.00234.x, 2007.

$\mathrm{Xu}, \mathrm{M}$. and Paul, M. R.: Covariant Lyapunov vectors of chaotic Rayleigh-Bénard convection, Phys. Rev. E, 93, 062208, https://doi.org/10.1103/PhysRevE.93.062208, 2016.
Yamada, M. and Ohkitani, K.: Lyapunov spectrum of a model of two-dimensional turbulence, Phys. Rev. Lett., 60, 983-986, https://doi.org/10.1103/PhysRevLett.60.983, 1988.

Yang, H.-L. and Radons, G.: Hydrodynamic Lyapunov modes and effective degrees of freedom of extended systems, J. Phys. A-Math. Theor., 46, 254015, https://doi.org/10.1088/17518113/46/25/254015, 2013. 